\title{
Two-Phase Flow in Wire Coating with Heat Transfer Analysis of an Elastic-Viscous Fluid
}

\author{
Zeeshan Khan, ${ }^{1,2}$ Rehan Ali Shah, ${ }^{3}$ Saeed Islam, ${ }^{1}$ and Bilal Jan ${ }^{2}$ \\ ${ }^{1}$ Department of Mathematics, Abdul Wali Khan University, Mardan, Khyber Pakhtunkhwa, Pakistan \\ ${ }^{2}$ Sarhad University of Science and Information Technology, Peshawar, Pakistan \\ ${ }^{3}$ Department of Mathematics, University of Engineering and Technology, Peshawar, Khyber Pakhtunkhwa, Pakistan \\ Correspondence should be addressed to Zeeshan Khan; zeeshansuit@gmail.com
}

Received 4 April 2016; Revised 12 May 2016; Accepted 27 June 2016

Academic Editor: Alexander Iomin

Copyright (C) 2016 Zeeshan Khan et al. This is an open access article distributed under the Creative Commons Attribution License, which permits unrestricted use, distribution, and reproduction in any medium, provided the original work is properly cited.

\begin{abstract}
This work considers two-phase flow of an elastic-viscous fluid for double-layer coating of wire. The wet-on-wet (WOW) coating process is used in this study. The analytical solution of the theoretical model is obtained by Optimal Homotopy Asymptotic Method (OHAM). The expression for the velocity field and temperature distribution for both layers is obtained. The convergence of the obtained series solution is established. The analytical results are verified by Adomian Decomposition Method (ADM). The obtained velocity field is compared with the existing exact solution of the same flow problem of second-grade fluid and with analytical solution of a third-grade fluid. Also, emerging parameters on the solutions are discussed and appropriate conclusions are drawn.
\end{abstract}

\section{Introduction}

The study of non-Newtonian fluids has gained deep attention from researchers due to its various applications in industries like oil, polymer, plastic, and so forth. Various models, both analytical and numerical, have been discussed in the study of non-Newtonian fluids. Fluids models are characterized by the underlying fluid grades like second grade, third grade, and so forth generalizing to $n$-grade fluids. In this study non-Newtonian third-grade fluids have been studied for their applicability in optical fiber coating. Third-grade fluids have been studied by many researchers. Siddiqui et al. [1] studied the torsion flow of such fluid. The same author studied heat flux of such fluids in two parallel plates which is discussed in [2]. Islam et al. [3] studied third-grade fluid with heat transfer. Aksoy and Pakdemirli [4] investigated the third-grade fluid flow in parallel plates with a porous medium.

The subject of two immiscible fluids flows with heat transfer has been briefly studied due to its importance in nuclear and chemical industries. It can be classified into three groups, namely, segregated flows, transitional or mixed flows, and dispersed flows [5]. Siddiqui et al. [6] studied two immiscible fluids in porous media. Batchelor [7] studied two immiscible fluids in an analogous plate. Two immiscible fluids have been extensively studied theoretically and experimentally $[8,9]$. Different types of fluids are used for wire and fiber optics coating, which depends upon the geometry of die, fluid viscosity, temperature of the wire or fiber optics, and the molten polymer.

Most relevant works on the wire and fiber optics coating are thus summarized in the following.

Shah et al. [10] studied the wire coating analysis with linearly varying temperature. Unsteady second-grade fluid with oscillating boundary condition inside the wire coating die was investigated by Shah et al. [11]. Exact solution was obtained for unsteady second-grade fluid in wire coating analysis [12]. Shah et al. [13] studied third-grade fluid with heat transfer in the wire coating analysis. All these attempts were related to single layer coating flow.

Immiscible fluid flow is used for many industrial and manufacturing processes such as oil industry or polymer production. Kim et al. [14] examined the theoretical prediction on the double-layer coating in wet-on-wet optical fiber coating process. Double-layer coating liquid flows were used by Kim and Kwak [15] in optical fiber manufacturing. For this purpose power-law fluid model was used. Recently Zeeshan et al. [16] used Phan Thien Tanner fluid in doublelayer optical fiber coating. The same author [17] investigated 


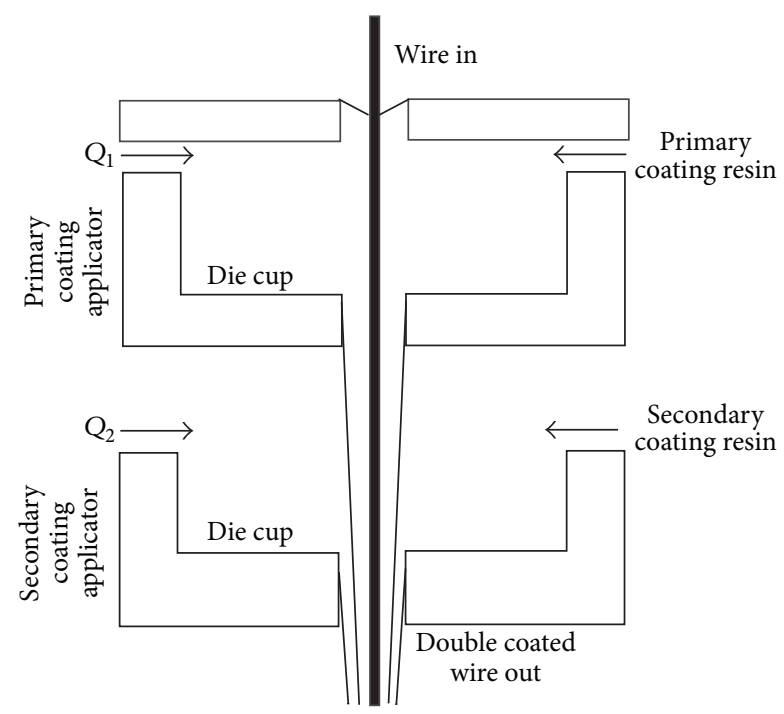

FIGURE 1: Manufacturing process of wire.

double-layer resin coating of optical fiber glass using wet-onwet coating process with constant pressure gradient. Twophase flow of an Oldroyd 8-constant fluid was used for optical fiber coating by Zeeshan et al. [18]. Flow and heat transfer in double-layer optical fiber coating using wet-on-wet coating process were investigated by Khan et al. [19].

Keeping in view the wide range of applications, an attempt is made to analyze the flow and heat transfer in two-phase flow of an elastic-viscous fluid in a pressure type die. In this paper, the task is to find the analytical solutions for the governing nonlinear equation arising in a coating metallic wire process inside a cylindrical roll die, to study the fluid flow behavior in particular, and to examine the effects of the non-Newtonian fluid parameters and axial distance from the center of the metallic wire. This is our first attempt to investigate the double-layer coating flow of an elastic-viscous fluid on the wire using wet-on-wet coating process. Apart from this, no one investigated the double-layer wire coating in wet-on-wet coating process using two immiscible elasticviscous fluids in a pressurized coating die. To the best of our knowledge, no such analysis of the double-layer coating flows of two immiscible elastic-viscous fluids on the wire is available in the literature.

The present paper is structured as follows. Section 2 is reserved for modeling of the problem. Solution of the problem is given in Section 3. Section 4 is reserved for analysis of results. Section 5 contains the concluding remarks.

\section{Modeling of the Problem}

The geometry of the problem is shown in Figure 1. The die and wire are concentric. The coordinate system is taken at the center of the wire, in which $r$ is taken perpendicular to the flow direction $z$.

The coating process is performed in two phases. In the first phase the uncovered wire of radius $R_{w}$ is dragged with constant velocity $V_{1}$ into the primary coating liquid. In the second phase the wet coating passes through the secondary coating die of radius $R_{d}$ and length $L$. In this way, the wire leaves the system with two layers of coating. The wet layers are dried up by ultraviolet (UV) lamps. The liquid parameters at each phase are generalized by corresponding phase number denoted by $j(j=1,2)$. The liquids are parameterized by temperature $\Theta^{(j)}$, the fluid density $\rho^{(j)}$, the viscosity $\mu^{(j)}$, thermal conductivity $K^{(j)}$, thermal expansion coefficient $\beta^{(j)}$, and the specific heat $c_{p}^{(j)}$. The gas velocity surrounding the polymer at the surface of coated wire is represented by $V_{2}$ as shown in Figure 2.

The governing equations for the two fluids are the continuity, momentum, and energy equations given as follows:

$$
\begin{aligned}
\nabla \cdot \widehat{u}^{(j)} & =0, \\
\rho^{(j)} \frac{D \widehat{u}^{(j)}}{D t} & =\nabla \cdot \mathbf{T}^{(j)}, \\
c_{p}^{(j)} \frac{D \Theta^{(j)}}{D t} & =k_{j} \nabla^{2} \Theta^{(j)}+\operatorname{tr}\left(\mathbf{T}^{(j)} \cdot \mathbf{L}\right),
\end{aligned}
$$

where $D / D t=\partial / \partial t+\widehat{u}^{(j)} \cdot \nabla$ is the substantive acceleration.

No-slip boundary conditions are taken at velocity. The temperature conditions $\widehat{\Theta}^{(1)}$ and $\widehat{\Theta}^{(2)}$ are taken at the fiber optics and die wall, respectively. At the fluid interface, we utilize the assumptions that the velocity, the shear stress, the pressure gradient, the temperature, and the heat flux are continuous.

The Cauchy stress $\mathbf{T}^{(j)}$ given in (2) is

$$
\mathbf{T}^{(j)}=-p \mathbf{I}+\mathbf{S}^{(j)} .
$$

In the above equation $p$ is the pressure and $\mathbf{I}$ and $\mathbf{S}^{(j)}$ are the identity and extra stress tensor, respectively.

For third-grade fluid $\mathbf{S}^{(j)}$ is defined as $[1-4,13]$

$$
\begin{aligned}
\mathbf{S}^{(j)}= & \mu^{(j)} \mathbf{A}_{1}^{(j)}+\alpha_{1}^{(j)} \mathbf{A}_{2}^{(j)}+\alpha_{2}^{(j)} \mathbf{A}_{1}^{(j)}+\tau_{1}^{(j)} \mathbf{A}_{2}^{(j)} \\
& +\tau_{2}^{(j)}\left(\mathbf{A}_{1}^{(j)} \mathbf{A}_{2}^{(j)}+\mathbf{A}_{2}^{(j)} \mathbf{A}_{1}^{(j)}\right)+\tau_{3}^{(j)}\left(\operatorname{tr} \mathbf{A}_{2}^{(j)}\right) \mathbf{A}_{1}^{(j)}
\end{aligned}
$$

In which $\alpha_{1}^{(j)}, \alpha_{2}^{(j)}, \tau_{1}^{(j)}, \tau_{2}^{(j)}$, and $\tau_{3}^{(j)}$ are constants and $\mathbf{A}_{1}^{(j)}$, $\mathbf{A}_{2}^{(j)}, \mathbf{A}_{3}^{(j)}$ are kinematic tensors defined by

$$
\begin{aligned}
& \mathbf{A}_{1}^{(j)}=\mathbf{L}^{(j) T}+\mathbf{L}^{(j)} \\
& \mathbf{A}_{n}^{(j)}=\mathbf{A}_{n-1}^{(j)} \mathbf{L}^{T}+\mathbf{L} \mathbf{A}_{n-1}^{(j)}+\frac{D \mathbf{A}_{n-1}^{(j)}}{D t}, \quad n=2,3
\end{aligned}
$$

where $T$ stands for transpose of a matrix.

For steady and unidirectional flow velocity, temperature and stress fields are defined as

$$
\begin{aligned}
& \widehat{u}^{(j)}=\left[0,0, w^{(j)}(r)\right], \\
& \Theta^{(j)}=\Theta^{(j)}(r), \\
& S^{(j)}=S^{(j)}(r) .
\end{aligned}
$$



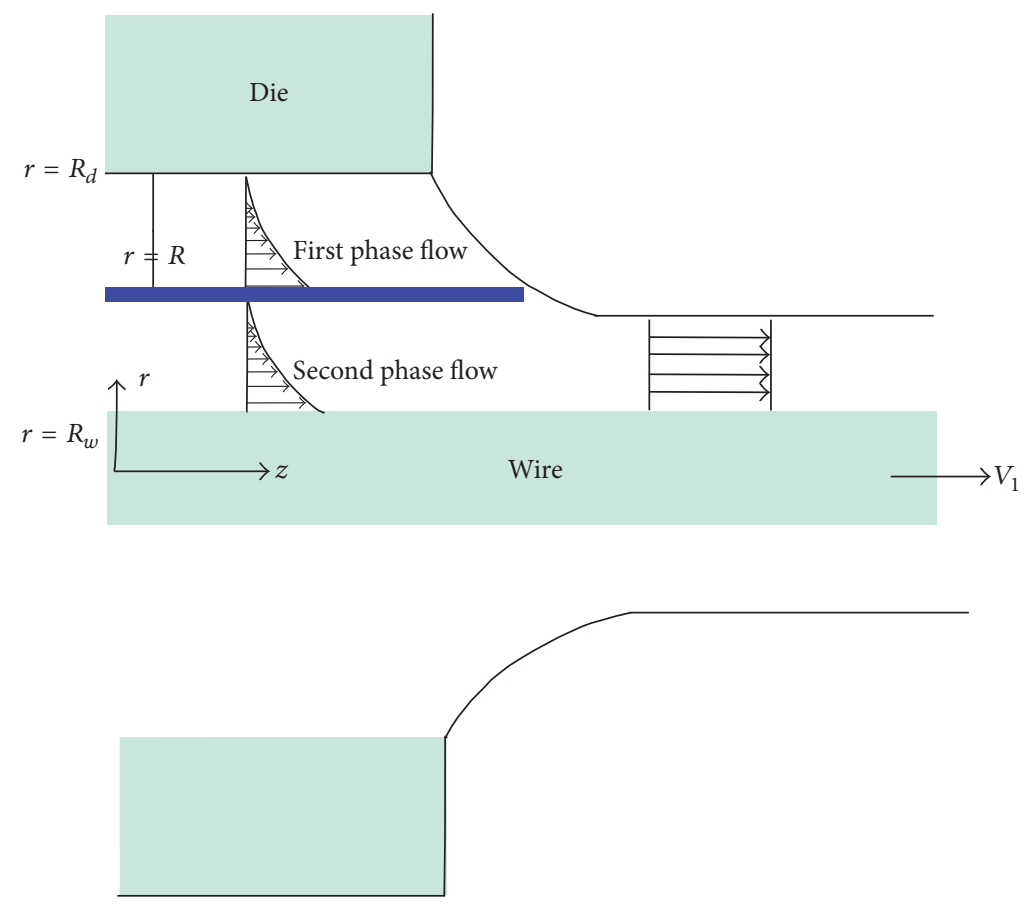

FIGURE 2: Two-phase flow model in wire coating die.

In view of (7), (1) is satisfied identically and from (2)-(6) we have

$$
\begin{aligned}
& S_{r r}^{(j)}=\left(2 \alpha_{1}^{(j)}+\alpha_{2}^{(j)}\right)\left(\frac{d w^{(j)}}{d r}\right)^{3}, \\
& S_{z z}^{(j)}=\alpha_{2}^{(j)}\left(\frac{d w^{(j)}}{d r}\right)^{2}, \\
& S_{r z}^{(j)}=\mu^{(j)} \frac{d w^{(j)}}{d r}+2\left(\tau_{2}^{(j)}+\tau_{3}^{(j)}\right)\left(\frac{d w^{(j)}}{d r}\right)^{3} .
\end{aligned}
$$

From (8), (2) and (3) reduce to

$$
\begin{aligned}
& 2\left(\tau_{2}^{(j)}+\tau_{3}^{(j)}\right) \frac{d}{d r}\left(r\left(\frac{d w^{(j)}}{d r}\right)^{3}\right) \\
& +\frac{\mu^{(j)}}{r} \frac{d}{d r}\left(r \frac{d w^{(j)}}{d r}\right)=0, \\
& K^{(j)}\left(\frac{d^{2}}{d r^{2}}+\frac{1}{r} \frac{d}{d r}\right) \Theta^{(j)}+\mu^{(j)}\left(\frac{d w^{(j)}}{d r}\right)^{2} \\
& \quad+2\left(\tau_{2}^{(j)}+\tau_{3}^{(j)}\right)\left(\frac{d w^{(j)}}{d r}\right)^{4}=0 .
\end{aligned}
$$

The pertinent boundary conditions on the velocity are [14-19]

$$
\begin{aligned}
& w^{(1)}=V_{1} \quad \text { at } r=R_{w}, \\
& w^{(2)}=0 \quad \text { at } r=R_{d}, \\
& w^{(1)}=w^{(2)}, \\
& S_{r z}^{(1)}=S_{r z}^{(2)} \\
& \quad \text { at } r=R .
\end{aligned}
$$

The pertinent boundary conditions on the temperature are [16-19]

$$
\begin{aligned}
\Theta^{(1)} & =\widehat{\Theta}^{(1)} \quad \text { at } r=R_{w}, \\
\Theta^{(2)} & =\widehat{\Theta}^{(2)} \text { at } r=R_{d}, \\
\Theta^{(1)} & =\Theta^{(2)}, \\
K^{(1)} \frac{d \Theta^{(1)}}{d r} & =K^{(2)} \frac{d \Theta^{(2)}}{d r} \\
& \text { at } r=R,
\end{aligned}
$$

(9) where (11) and (13) are the interface conditions on velocity and temperature, respectively.

The volume flow rate at some control surface is

$$
Q^{(j)}=\pi V_{1}\left(h^{(j) 2}-R_{w}^{2}\right),
$$

where $h^{(j)}$ is the radius of the coated wire. 
The volume flow rate is [14-19]

$$
Q^{(j)}=\int_{R_{w}}^{R_{d}} r w^{(j)}(r) d r .
$$

Thickness of the coating wire is [14-19]

$$
\begin{aligned}
h^{(1)} & =R_{w}\left(\left(1+\frac{Q^{(1)}}{\pi V_{1} R_{w}^{2}}\right)-1\right) \\
h^{(2)} & =R_{w}\left(\left[\left(1+\frac{h^{(1)}}{R_{w}}\right)^{2}+\frac{Q^{(2)}}{\pi V_{1} R_{w}^{2}}\right]^{1 / 2}\right. \\
& \left.+\left(1+\frac{h^{(1)}}{R_{w}}\right)\right) .
\end{aligned}
$$

In view of (17), (9)-(16) can be reduced to the following set of nondimensional equations, respectively:

$$
\begin{aligned}
& r^{*}=\frac{r}{R_{w}}, \\
& w_{j}^{*}=\frac{w^{(j)}}{V_{1}}, \\
& \theta_{j}^{*}=\frac{\Theta^{(j)}-\widehat{\Theta}^{(2)}}{\widehat{\Theta}^{(2)}-\widehat{\Theta}^{(1)}}, \\
& \tau_{0}^{(j)}=\tau_{2}^{(j)}+\tau_{3}^{(j)}, \\
& \frac{R_{d}}{R_{w}}=\delta>1, \\
& \frac{R}{R_{w}}=\Omega, \\
& \frac{V_{2}}{V_{1}}=U \text {, } \\
& \lambda=\frac{\mu^{(2)}}{\mu^{(1)}}, \\
& K=\frac{K^{(2)}}{K^{(1)}}, \\
& B r_{j}=\frac{\mu^{(j)} V_{1}^{2}}{K^{(j)}\left(\widehat{\Theta}^{(2)}-\widehat{\Theta}^{(1)}\right)}, \\
& \beta_{j}=\frac{\tau_{0}^{(j)}}{\mu^{(j)}\left(R_{w}^{2} / V_{1}^{2}\right)} .
\end{aligned}
$$

$$
\begin{gathered}
r \frac{d^{2} w_{j}}{d r^{2}}+\frac{d w_{j}}{d r}+2 \beta_{j}\left(3 r \frac{d^{2} w_{j}}{d r^{2}}\left(\frac{d w_{j}}{d r}\right)^{2}\right. \\
\left.+\left(\frac{d w_{j}}{d r}\right)^{3}\right)=0
\end{gathered}
$$

$$
\begin{aligned}
& \frac{d^{2} \theta_{j}}{d r^{2}}+\frac{1}{r} \frac{d \theta_{j}}{d r}+B r_{j}\left(\frac{d w_{j}}{d r}\right)^{2}+2 B r_{j} \beta_{j}\left(\frac{d w_{j}}{d r}\right)^{4} \\
& \quad=0 \\
& w_{1}(1)=1 \\
& w_{2}(\delta)=U, \\
& w_{1}=w_{2}, \\
& S_{r z}^{(1)}=S_{r z}^{(2)}
\end{aligned}
$$
at $r=\Omega$,

$$
\theta_{1}(1)=0 \text {, }
$$$$
\theta_{2}(\delta)=1 \text {, }
$$

$\theta_{1}=\theta_{2}$

$$
\frac{d \theta_{1}}{d r}=\frac{K}{\delta} \frac{d \theta_{2}}{d r}
$$

$$
\begin{aligned}
Q_{j} & =Q_{1}+Q_{2}=\frac{Q^{(j)}}{2 \pi R_{w}^{2} V_{1}}=\int_{1}^{\Omega} r w_{1}(r)(r) d r \\
& +\int_{\Omega}^{\delta} r w_{2}(r)(r) d r,
\end{aligned}
$$

$$
\begin{aligned}
h_{j} & =h_{1}+h_{2}=\frac{h^{(j)}}{R_{w}}=\left(\left[1+2 \int_{1}^{\Omega} r w_{1}(r)(r) d r\right]^{1 / 2}\right. \\
& -1)+\left(\left[\left(1+h_{1}\right)^{2}+2 \int_{\Omega}^{\delta} r w_{2}(r)(r) d r\right]^{1 / 2}\right. \\
& \left.+\left(1+h_{1}\right)\right) .
\end{aligned}
$$

\section{Solution of the Problem}

The OHAM is a steadfast method which has been broadly used by the researchers to solve nonlinear problems. One special area of application of this method is to solve equations arising when non-Newtonian fluids [20-25] are studied. To solve (18) corresponding to the boundary conditions given in (20) and (21), we apply OHAM and ADM [26-28]. The details of OHAM and ADM are given in Appendix. Here, only the OHAM solution is given. 
By using OHAM, the zeroth-, first-, and second-order solutions for both layers are given below:

$$
\begin{aligned}
w_{(1) 0}= & \sigma_{1}+\sigma_{2} \ln r \\
w_{(2) 0}= & \sigma_{3}+\sigma_{4} \ln r \\
w_{(1) 1}= & \sigma_{5}+\sigma_{6} \ln r-r k_{2}-C_{1} k_{2} \ln r+\frac{1}{8} \frac{k_{2}^{2} \beta_{1}}{r^{4}} \\
& -\frac{1}{2} \frac{C_{1} k_{2}^{2} \beta_{1}}{r^{2}}, \\
w_{(2) 1}= & \sigma_{7}+\sigma_{8} \ln r-r k_{2}-C_{3} k_{2} \ln r+\frac{1}{8} \frac{k_{2}^{2} \beta_{2}}{r^{4}} \\
& -\frac{1}{2} \frac{C_{3} k_{2}^{2} \beta_{2}}{r^{2}},
\end{aligned}
$$$$
w_{(1) 2}=\sigma_{9}+\sigma_{10} \ln r-2 \ln r C_{1} k_{1}-2 \ln r C_{1}^{2} k_{1}+r k_{2}
$$$$
-\frac{C_{1} k_{1}}{r}-r C_{1} k_{2}-\frac{C_{1}^{2} k_{2}}{r}+\frac{1}{5} \frac{C_{1} k_{2}^{2} \beta_{1}}{r^{5}}
$$$$
-\frac{1}{3} \frac{C_{1} k_{2}^{2} \beta_{1}}{r^{3}}-\frac{C_{1} k_{2}^{2} \beta_{1}}{4 r^{2}}+\frac{C_{1}^{2} k_{2}^{2} \beta_{1}}{10 r^{5}}
$$$$
-\frac{C_{1}^{2} k_{2}^{2} \beta_{1}}{32 r^{4}}-\frac{C_{1}^{2} k_{2}^{2} \beta_{1}}{3 r^{3}}+\frac{C_{1}^{2} k_{2}^{2} \beta_{1}}{4 r^{2}}-\frac{C_{2} k_{2}^{2} \beta_{1}}{r^{2}}
$$$$
+\frac{3 C_{1}^{2} k_{1} k_{2}^{2} \beta_{1}}{r^{2}}+\frac{6 C_{1} k_{2}^{3} \beta_{1}}{r}-\frac{\sigma_{4}}{r}-\frac{C_{1}^{2} k_{2}^{4} \beta_{1}^{2}}{2 r^{2}}
$$$$
-\frac{C_{1}^{2} k_{2}^{4} \beta_{1}^{2}}{3 r^{6}}-\frac{3 C_{1}^{2} k_{2}^{4} \beta_{1}^{2}}{2 r^{4}}-\frac{3 C_{1} k_{2}^{2} \beta_{1} \sigma_{4}}{r^{2}}
$$$$
-\sigma_{4} \ln r+C_{1} \sigma_{4} \ln r,
$$$$
w_{(2) 2}=\sigma_{11}+\sigma_{12} \ln r-2 \ln r C_{3} k_{2}-2 \ln r C_{3}^{2} k_{1}+r k_{2}
$$$$
-\frac{C_{3} k_{1}}{r}-r C_{1} k_{2}-\frac{C_{3}^{2} k_{2}}{r}+\frac{1}{5} \frac{C_{1} k_{2}^{2} \beta_{2}}{r^{5}}
$$$$
-\frac{1}{3} \frac{C_{3} k_{2}^{2} \beta_{2}}{r^{3}}-\frac{C_{3} k_{2}^{2} \beta_{2}}{4 r^{2}}+\frac{C_{3}^{2} k_{2}^{2} \beta_{2}}{10 r^{5}}
$$$$
-\frac{C_{3}^{2} k_{2}^{2} \beta_{2}}{32 r^{4}}-\frac{C_{3}^{2} k_{2}^{2} \beta_{2}}{3 r^{3}}+\frac{C_{3}^{2} k_{2}^{2} \beta_{2}}{4 r^{2}}-\frac{C_{4} k_{2}^{2} \beta_{2}}{r^{2}}
$$$$
+\frac{3 C_{3}^{2} k_{1} k_{2}^{2} \beta_{2}}{r^{2}}+\frac{6 C_{3} k_{2}^{3} \beta_{2}}{r}-\frac{\sigma_{6}}{r}-\frac{C_{3}^{2} k_{2}^{4} \beta_{2}^{2}}{2 r^{2}}
$$$$
-\frac{C_{3}^{2} k_{2}^{4} \beta_{2}^{2}}{3 r^{6}}-\frac{3 C_{3}^{2} k_{2}^{4} \beta_{2}^{2}}{2 r^{4}}-\frac{3 C_{3} k_{2}^{2} \beta_{2} \sigma_{6}}{r^{2}}
$$$$
-\sigma_{6} \ln r+C_{3} \sigma_{6} \ln r \text {. }
$$

Collecting the results, we write the velocity field obtained by OHAM up to second-order approximation as

$$
\begin{aligned}
& w_{1}(r)=w_{(1) 0}(r)+w_{(1) 1}\left(r, C_{1}\right)+w_{(1) 2}\left(r, C_{1}, C_{2}\right) \\
& +\cdots, \\
& w_{2}(r)=w_{(2) 0}(r)+w_{(2) 1}\left(r, C_{1}\right)+w_{(2) 2}\left(r, C_{3}, C_{4}\right) \\
& +\cdots .
\end{aligned}
$$

The series solution up to second-order approximation for both layers is

$$
\begin{aligned}
w_{1}= & \Phi_{11}+\Phi_{12} \ln r+r \Phi_{13}+\frac{1}{r} \Phi_{14}+\frac{1}{r^{2}} \Gamma_{15}+\frac{1}{r^{3}} \Phi_{16} \\
& +\frac{1}{r^{4}} \Phi_{17}+\frac{1}{r^{5}} \Phi_{18}, \\
w_{2}= & \Gamma_{11}+\Gamma_{12} \ln r+r \Gamma_{13}+\frac{1}{r} \Gamma_{14}+\frac{1}{r^{2}} \Gamma_{15}+\frac{1}{r^{3}} \Gamma_{16} \\
& +\frac{1}{r^{4}} \Gamma_{17}+\frac{1}{r^{5}} \Gamma_{18} .
\end{aligned}
$$

In view of (24) the volume flow rate in each phase is obtained as follows:

$$
\begin{aligned}
Q_{1} & \frac{1}{4}+\Phi_{14}(\Omega-1)+\Phi_{11}\left(\Omega^{2}-1\right)-\frac{\Phi_{12}}{4} \Omega^{2} \\
& +\frac{\Phi_{13}}{3}\left(\Omega^{3}-1\right)+\left(\frac{\Phi_{12}}{3} \Omega^{2}+\Phi_{15}\right) \ln \Omega \\
& -\Phi_{16}\left(\frac{1}{\Omega}-1\right)-\frac{\Phi_{17}}{2}\left(\frac{1}{\Omega^{2}}-1\right) \\
& -\frac{\Phi_{18}}{3}\left(\frac{1}{\Omega^{3}}-1\right), \\
Q_{2} & (\Omega-\delta)\left(\left(\frac{\Gamma_{11}}{2}(\Omega+\delta)+\frac{\Gamma_{13}}{2}(\Omega+\delta)^{2}+\Gamma_{14}\right)\right) \\
& +\Gamma_{15}\left(\ln \ln \left(\frac{\Omega}{\delta}\right)\right) \\
& -\left(\frac{1}{\Omega}-\frac{1}{\delta}\right) \Gamma_{16} \frac{\Gamma_{11}}{2}\left(\frac{1}{\Omega}+\frac{1}{\delta}\right) \\
& +\frac{\Gamma_{86}}{2}\left(\frac{1}{\Omega}+\frac{1}{\delta}\right)^{2} .
\end{aligned}
$$




\section{Solution for the Temperature Distribution}

Now inserting $w_{1}(r)$ and $w_{2}(r)$ from (28) into (19) and solving with boundary conditions given in (22) and (23), we obtain the temperature fields for both layers as

$$
\begin{aligned}
& \theta_{1}= c_{1}+c_{2} \ln r+\frac{\Psi_{1}}{r^{22}}+\frac{\Psi_{2}}{r^{21}}+\frac{\Psi_{3}}{r^{20}}+\frac{\Psi_{4}}{r^{19}}+\frac{\Psi_{5}}{r^{18}}+\frac{\Psi_{6}}{r^{17}} \\
&+\frac{\Psi_{7}}{r^{16}}+\frac{\Psi_{8}}{r^{15}}+\frac{\Psi_{9}}{r^{14}}+\frac{\Psi_{10}}{r^{13}}+\frac{\Psi_{11}}{r^{12}}+\frac{\Psi_{12}}{r^{11}} \\
&+\frac{\Psi_{13}}{r^{10}}+\frac{\Psi_{14}}{r^{9}}+\frac{\Psi_{15}}{r^{8}}+\frac{\Psi_{16}}{r^{7}}+\frac{\Psi_{17}}{r^{6}}+\frac{\Psi_{18}}{r^{5}} \\
&+\frac{\Psi_{19}}{r^{4}}+\frac{\Psi_{20}}{r^{3}}+\frac{\Psi_{21}}{r^{2}}+\frac{\Psi_{22}}{r}+r \Psi_{23}+r^{2} \Psi_{24} \\
&+\Psi_{25}(\ln r)^{2}, \\
& \theta_{2}= c_{3}+c_{4} \ln r+\frac{\Lambda_{1}}{r^{22}}+\frac{\Lambda_{2}}{r^{21}}+\frac{\Lambda_{3}}{r^{20}}+\frac{\Lambda_{4}}{r^{19}}+\frac{\Lambda_{5}}{r^{18}} \\
&+\frac{\Lambda_{6}}{r^{17}}+\frac{\Lambda_{7}}{r^{16}}+\frac{\Lambda_{8}}{r^{15}}+\frac{\Lambda_{9}}{r^{14}}+\frac{\Lambda_{10}}{r^{13}}+\frac{\Lambda_{11}}{r^{12}} \\
&+\frac{\Lambda_{12}}{r^{11}}+\frac{\Lambda_{13}}{r^{10}}+\frac{\Lambda_{14}}{r^{9}}+\frac{\Lambda_{15}}{r^{8}}+\frac{\Lambda_{16}}{r^{7}}+\frac{\Lambda_{17}}{r^{6}} \\
&+\frac{\Lambda_{18}}{r^{5}}+\frac{\Lambda_{19}}{r^{4}}+\frac{\Lambda_{20}}{r^{3}}+\frac{\Lambda_{21}}{r^{2}}+\frac{\Lambda_{22}}{r}+r \Lambda_{23} \\
&+r^{2} \Lambda_{24}+\Lambda_{25}(\ln r)^{2},
\end{aligned}
$$

where $c_{1}, c_{2}, c_{3}, c_{4}, \sigma_{1}, \sigma_{2}, \sigma_{3}, \sigma_{4}, \sigma_{5}, \sigma_{6}, \sigma_{7}, \sigma_{8}, \sigma_{9}, \sigma_{10}, \sigma_{11}$, $\sigma_{12}, \Psi_{11}, \Psi_{12}, \Psi_{13}, \Psi_{14}, \Psi_{15}, \Psi_{16}, \Psi_{17}, \Psi_{18}, \Gamma_{11}, \Gamma_{12}, \Gamma_{13}, \Gamma_{14}, \Gamma_{15}$, $\Gamma_{16}, \Gamma_{17}, \Gamma_{18}, \Lambda_{1}, \Lambda_{2}, \Lambda_{3}, \Lambda_{4}, \Lambda_{5}, \Lambda_{6}, \Lambda_{7}, \Lambda_{8}, \Lambda_{9}, \Lambda_{10}, \Lambda_{11}$, $\Lambda_{12}, \Lambda_{13}, \Lambda_{14}, \Lambda_{15}, \Lambda_{16}, \Lambda_{17}, \Lambda_{18}, \Lambda_{19}, \Lambda_{20}, \Lambda_{21}, \Lambda_{22}, \Lambda_{23}$, and $\Lambda_{24}$ are all constants containing the convergence-control parameters $C_{1}, \ldots, C_{4}$, which are optimally determined by the method of least squares and are given in Appendix.

\section{Analysis of the Results}

Two-phase flow of an elastic-viscous third-grade fluid is used for wire coating. Actually, we are making a platform for coating of polymer on the wire using theoretical approach. The wire needs flexibility; that is why we need two-layer coating of the polymer. The inner coating or primary coating protects the wire from bending, while the outer coating or secondary coating protects the primary coating from mechanical damage. For coating of the double-layer wire the wet-on-wet coating process is applied. Axial velocity distribution, flow rate, thickness of the coated wire, and temperature distributions for each phase are obtained by OHAM.

The convergence of the method is also necessary to check the reliability of the methodology. The convergence of the obtained series is shown in Figures 3 and 4. The current computed results obtained are also compared with ADM, and an outstanding correspondence is seen to exist between the two sets of data as revealed in Figures 5 and 6 and Table 1. Furthermore, the obtained results are also compared with
TABLE 1: Numerical comparison of OHAM and ADM when $\beta_{1}=$ $0.1, \beta_{2}=0.5, \lambda=0.3, \delta=2, C_{1}=-0.001243429, C_{2}=$ $-0.000248121, C_{3}=0.001032981$, and $C_{4}=0.000294127$.

\begin{tabular}{lccc}
\hline$r$ & OHAM & ADM & Absolute error \\
\hline 1 & 1 & 1 & 0 \\
1.1 & 0.031721271 & 0.031621270 & $0.136 \times 10^{-7}$ \\
1.2 & 0.022015241 & 0.022025243 & $0.251 \times 10^{-7}$ \\
1.3 & 0.006210390 & 0.006230392 & $0.872 \times 10^{-7}$ \\
1.4 & 0.011607241 & 0.011606221 & $0.101 \times 10^{-6}$ \\
1.5 & 0.010442045 & 0.010442141 & $0.712 \times 10^{-7}$ \\
1.6 & 0.001520519 & 0.001522512 & $0.101 \times 10^{-7}$ \\
1.7 & 0.006014981 & 0.007214980 & $0.106 \times 10^{-7}$ \\
1.8 & 0.004101612 & 0.004100632 & $0.103 \times 10^{-7}$ \\
1.9 & 0.000032263 & 0.000031264 & $0.120 \times 10^{-7}$ \\
2.0 & $0.003051 \times 10^{-19}$ & $0.003061 \times 10^{-19}$ & $0.011 \times 10^{-20}$ \\
\hline
\end{tabular}

TABLE 2: Comparison of the present work with published work [12] when $\beta_{1}=0.1, \beta_{2}=0.5, \lambda=0.3, \delta=2, C_{1}=-0.001243429, C_{2}=$ $-0.000248121, C_{3}=0.001032981$, and $C_{4}=0.000294127$.

\begin{tabular}{lccc}
\hline$r$ & Present work & Published work [12] & Absolute error \\
\hline 1 & 1 & 1 & 0 \\
1.1 & 0.040810 & 0.040825 & 0.000005 \\
1.2 & 0.043001 & 0.043004 & 0.000003 \\
1.3 & 0.040173 & 0.040103 & 0.000007 \\
1.4 & 0.032718 & 0.032712 & 0.000006 \\
1.5 & 0.021556 & 0.021551 & 0.000005 \\
1.6 & 0.003731 & 0.003721 & 0.000003 \\
1.7 & 0.008221 & 0.008220 & 0.000001 \\
1.8 & 0.006013 & 0.006011 & 0.000002 \\
1.9 & 0.000012 & 0.000022 & 0.000010 \\
2.0 & 0.000010 & 0.000010 & 0 \\
\hline
\end{tabular}

TABLE 3: Comparison of the present work with published work [13] when $\beta_{1}=1=\beta_{2}, \lambda=0.3, \delta=2, C_{1}=-0.001243429, C_{2}=$ $-0.000248121, C_{3}=0.001032981$, and $C_{4}=0.000294127$.

\begin{tabular}{lccc}
\hline$r$ & Present work & Published work [13] & Absolute error \\
\hline 1.0 & 1 & 1 & 0 \\
1.1 & 0.251392 & 0.251080 & 0.000312 \\
1.2 & 0.273548 & 0.273526 & 0.000022 \\
1.3 & 0.130913 & 0.130915 & 0.000012 \\
1.4 & 0.193270 & 0.193260 & 0.000010 \\
1.5 & 0.192713 & 0.1932707 & 0.000512 \\
1.6 & 0.093035 & 0.0930262 & 0.000088 \\
1.7 & 0.027517 & 0.0275210 & 0.000004 \\
1.8 & 0.0122612 & 0.0122611 & 0.0000001 \\
1.9 & 0.0000231 & 0.0000232 & 0.0000001 \\
2.0 & 0 & 0 & 0 \\
\hline
\end{tabular}

preceding published related literature, as a special case of the problem and admirable agreement is observed in this case also, as shown in Tables 2 and 3. 


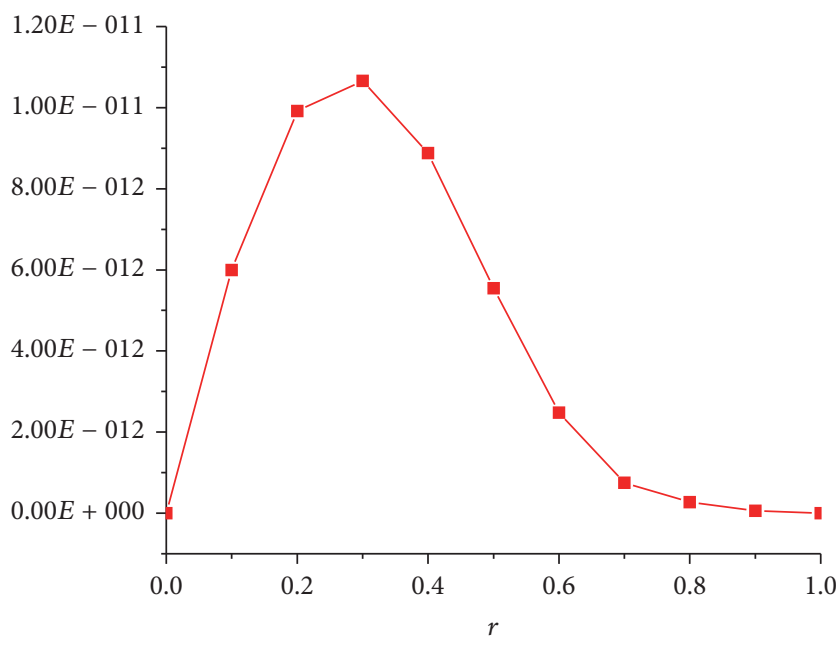

FIGURE 3: Error graph (showing convergence of OHAM): OHAM up to second-order solution when $U=0.2, \beta_{1}=0.3, \beta_{2}=1, \lambda=0.3$, and $\delta=2$.

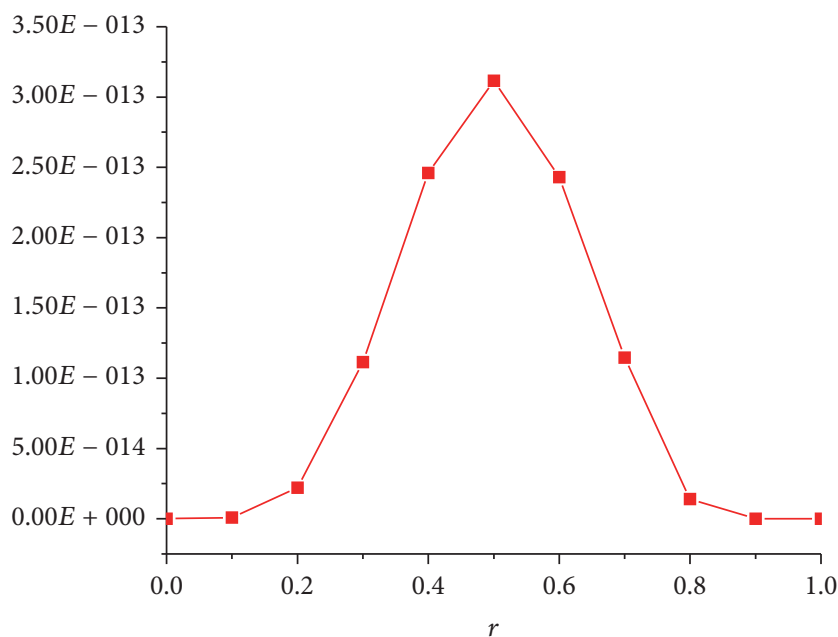

FIGURE 4: Error graph (showing convergence of OHAM): OHAM up to second-order solution when $U=0.2, \beta_{1}=0.1, \beta_{2}=0.3, \lambda=0.3$, and $\delta=2$.

The variation of the non-Newtonian parameter $\beta_{1}$, the Brinkman number $B r_{1}$, the conductivity ratio $K$, and the radii ratio $\delta$ on the velocity, temperature, and thickness of coated wire are elaborated numerically in Tables $4-8$. Table 4 shows the effects of the non-Newtonian parameter $\beta_{1}$ on the velocity profile. Here, we varied $\beta_{1}=0.2,0.3,0.5,0.8$ and fixed the values of $U=0.2, \lambda=0.1, \beta_{2}=0.5$, and $\delta=2$. It is to be noted that the rise of the non-Newtonian parameter decreases the speed of the flow. The effects of the Brinkman number $B r_{1}$ and the conductivity ratio $K$ on the temperature distribution are given in Tables 5 and 6. In Table 5 we varied $B r_{1}=5,10,13,18$ and fixed the values of $\lambda=0.1, \beta_{1}=0.1, \beta_{2}=0.3$, and $\delta=2$. Table 6 gives the numerical values of the temperature distribution by taking different values of the conductivity ratio $K$. We observe from these tables that the temperature inside the fluid increases by increasing the values of the

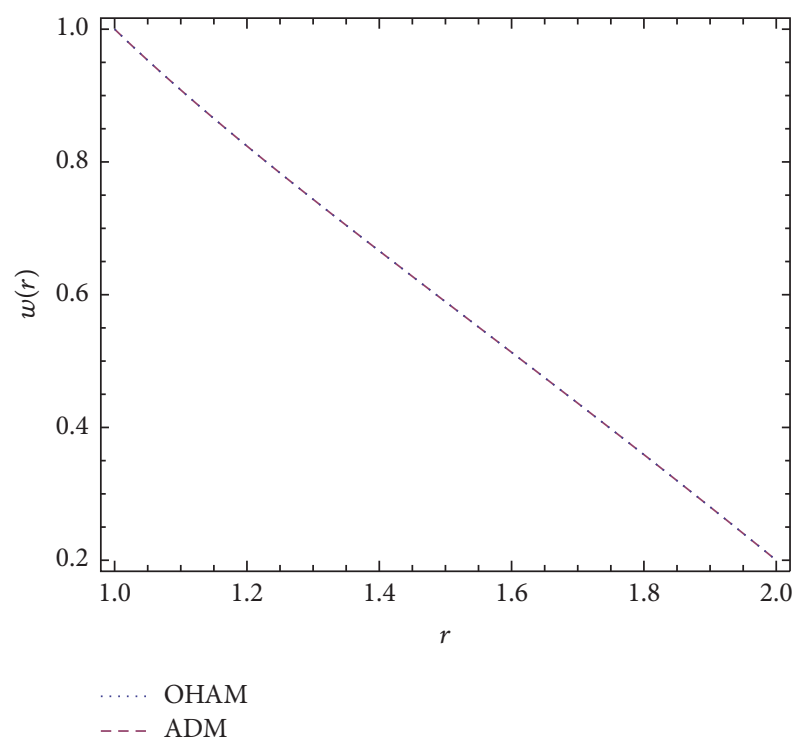

FIGURE 5: Velocity comparison between OHAM and ADM results when $U=0.2, \beta_{1}=0.1, \beta_{2}=0.5, \lambda=0.3, C_{1}=-0.001243429$, $C_{2}=-0.000248121, C_{3}=0.001032981$, and $C_{4}=0.000294127$.

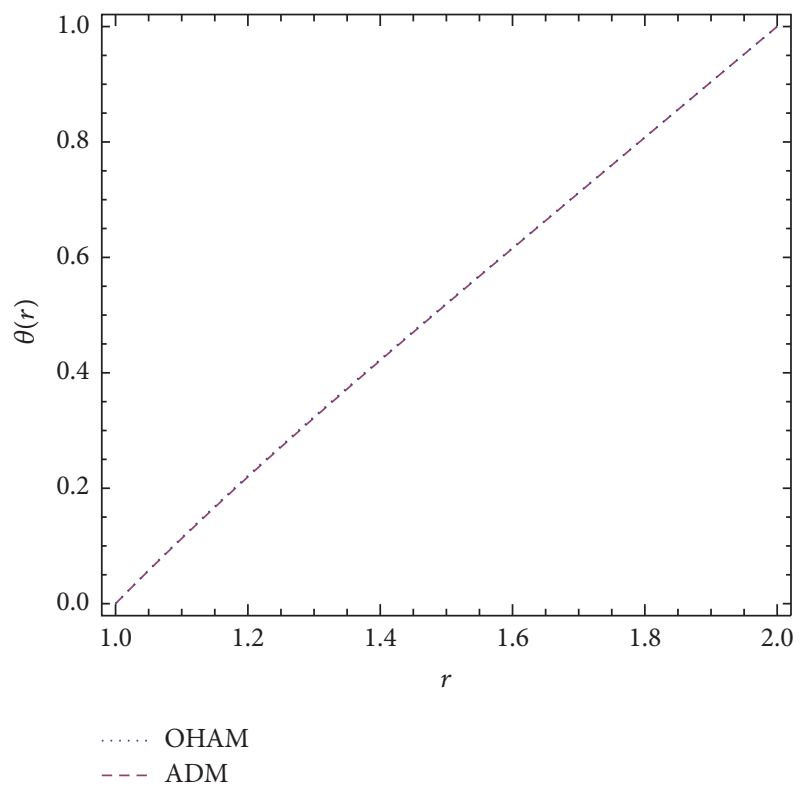

FIGURE 6: Temperature comparison between OHAM and ADM results when $\beta_{1}=0.1, \beta_{2}=0.5, \lambda=0.3, B r_{1}=3, B r_{2}=5, \delta=2$, $C_{1}=-0.001243429, C_{2}=-0.000248121, C_{3}=0.001032981$, and $C_{4}=0.000294127$.

conductivity ratio. The increase in the Brinkman number significantly affects the temperature distribution as shown in Table 5. Furthermore, the temperature profile attains its maximum values at the center of the annular die for different values of $B r_{1}$; then it decreases to meet the far field boundary conditions for fixed parameters. Tables 7 and 8 present the impact of enlarging the radii ratio $\delta$ and the viscosity ratio $\lambda$ on the thickness of the coated wire, respectively. From these 
TABLE 4: Velocity profile for various values of $\beta_{1}$, when $\beta_{2}=0.2, \lambda=$ $0.3, \delta=2, C_{1}=-0.001243429, C_{2}=-0.000248121, C_{3}=$ 0.001032981 , and $C_{4}=0.000294127$.

\begin{tabular}{lcccc}
\hline$r$ & $\beta_{1}=0.2$ & $\beta_{1}=0.3$ & $\beta_{1}=0.5$ & $\beta_{1}=0.8$ \\
\hline 1.0 & 1 & 1 & 1 & 1 \\
1.1 & 0.95913 & 0.925982 & 0.895634 & 0.868704 \\
1.2 & 0.91910 & 0.859577 & 0.807062 & 0.761416 \\
1.3 & 0.87837 & 0.798656 & 0.730893 & 0.673223 \\
1.4 & 0.83392 & 0.738489 & 0.660351 & 0.595324 \\
1.5 & 0.78239 & 0.674541 & 0.589529 & 0.520467 \\
1.6 & 0.71988 & 0.602079 & 0.512754 & 0.442056 \\
1.7 & 0.64172 & 0.515892 & 0.424158 & 0.353576 \\
1.8 & 0.54245 & 0.410078 & 0.317355 & 0.248183 \\
1.9 & 0.41560 & 0.277826 & 0.185196 & 0.118392 \\
2.0 & 0 & 0 & 0 & 0 \\
\hline
\end{tabular}

TABLE 5: Temperature distributions for several values of $B r_{1}$, when $\beta_{1}=0.1, \beta_{2}=0.3, B r_{2}=10, K=0.3, \lambda=0.3, \delta=2, C_{1}=$ $-0.001243429, C_{2}=-0.000248121, C_{3}=0.001032981$, and $C_{4}=$ 0.000294127 .

\begin{tabular}{lcccc}
\hline$r$ & $B r_{1}=5$ & $B r_{1}=10$ & $B r_{1}=13$ & $B r_{1}=18$ \\
\hline 1.0 & 0 & 0 & 0 & 0 \\
1.1 & 2.98376 & 4.93489 & 7.91381 & 12.11141 \\
1.2 & 4.03495 & 7.06917 & 11.45326 & 17.76048 \\
1.3 & 4.54514 & 7.95893 & 12.19506 & 18.50829 \\
1.4 & 4.68145 & 8.06743 & 12.69515 & 20.07146 \\
1.5 & 4.75303 & 8.28599 & 13.05673 & 20.05669 \\
1.6 & 4.23232 & 7.24325 & 10.65331 & 15.84121 \\
1.7 & 3.76761 & 6.24576 & 8.62779 & 12.39587 \\
1.8 & 3.19079 & 5.03953 & 6.25438 & 8.39888 \\
1.9 & 2.52216 & 2.18318 & 3.62751 & 4.00935 \\
2.0 & 1 & 1 & 1 & 1 \\
\hline
\end{tabular}

TABLE 6: Temperature distributions for several values of conductivity ratio $K$, when $\beta_{1}=0.1, \beta_{2}=0.3, B r_{1}=10, B r_{2}=15, \lambda=$ $0.3, \delta=2, C_{1}=-0.001243429, C_{2}=-0.000248121, C_{3}=$ 0.001032981 , and $C_{4}=0.000294127$.

\begin{tabular}{lcccc}
\hline$r$ & $K=0.1$ & $K=0.3$ & $K=0.7$ & $K=1$ \\
\hline 1.0 & 0 & 0 & 0 & 0 \\
1.1 & 0.179451 & 0.152275 & 0.132929 & 0.119676 \\
1.2 & 0.318216 & 0.278081 & 0.249429 & 0.229723 \\
1.3 & 0.430445 & 0.386476 & 0.352996 & 0.333271 \\
1.4 & 0.526147 & 0.483906 & 0.453590 & 0.432591 \\
1.5 & 0.612117 & 0.574786 & 0.547935 & 0.529281 \\
1.6 & 0.692744 & 0.661999 & 0.639849 & 0.624425 \\
1.7 & 0.770691 & 0.473284 & 0.730476 & 0.718723 \\
1.8 & 0.847449 & 0.831795 & 0.820495 & 0.812607 \\
1.9 & 0.923776 & 0.915937 & 0.910277 & 0.906235 \\
2.0 & 1 & 1 & 1 & 1 \\
\hline
\end{tabular}

tables it is observed that the thickness of coated wire increases with increase of $\delta$ and $\lambda$, respectively.
TABLE 7: Thickness of coated wire for various values of $\delta$ when $\beta_{1}=0.1, \beta_{2}=0.3, \lambda=0.3, C_{1}=-0.001243429, C_{2}=$ $-0.000248121, C_{3}=0.001032981$, and $C_{4}=0.000294127$.

\begin{tabular}{lcccc}
\hline$r$ & $\delta=1.2$ & $\delta=1.4$ & $\delta=1.8$ & $\delta=2$ \\
\hline 1.0 & 0.826354 & 1.22604 & 1.65398 & 1.89453 \\
1.2 & 0.997462 & 1.52918 & 1.85637 & 2.362485 \\
1.4 & 1.032548 & 1.983550 & 2.482702 & 3.004536 \\
1.6 & 1.557209 & 2.0047563 & 3.762449 & 3.978162 \\
1.8 & 1.982107 & 2.8834252 & 4.772954 & 4.984535 \\
2.0 & 2.452093 & 3.962949 & 5.035244 & 5.783529 \\
\hline
\end{tabular}

TABLE 8: Thickness of coated wire for various values of $\lambda$ when $\beta_{1}=0.1, \beta_{2}=0.3, \delta=2, C_{1}=-0.001243429, C_{2}=$ $-0.000248121, C_{3}=0.001032981$, and $C_{4}=0.000294127$.

\begin{tabular}{lcccc}
\hline$r$ & $\lambda=0.2$ & $\lambda=0.4$ & $\lambda=0.5$ & $\lambda=0.6$ \\
\hline 1.0 & 1.25475 & 1.43402 & 1.74536 & 2.25475 \\
1.2 & 1.55389 & 2.23425 & 3.45322 & 3.88452 \\
1.4 & 2.37628 & 2.54763 & 3.89325 & 4.01045 \\
1.6 & 3.00124 & 3.24564 & 4.08439 & 4.745385 \\
1.8 & 3.84735 & 4.10235 & 4.47365 & 5.543343 \\
2.0 & 4.362448 & 4.87524 & 5.014326 & 6.342650 \\
\hline
\end{tabular}

Additionally, the thickness of the coated wire can be maintained at a required level by adjusting these parameters.

\section{Conclusions}

Two-phase flow of an elastic-viscous third-grade fluid and wet-on-wet coating process is applied for wire coating analysis. The obtained nonlinear equations are solved for velocity fields and temperature distribution by OHAM. ADM is also used for clarity. The effect of various emerging parameters on the velocity profile, thickness of coated wire, and temperature distribution is discussed numerically. It is observed that, with increasing $\beta_{1}$, the velocity of the fluid decreases. The temperature inside the fluid is found to be increased with increasing the Brinkman number $B r_{1}$ and conductivity ratio $K$. Furthermore, the thickness of the coated wire also increases with $\delta$ and $\lambda$.

\section{Appendix}

\section{A. Analysis of ADM}

$\mathrm{ADM}$ is an analytical technique for decomposing an unknown function into infinitely many components. For better understanding we consider the following:

$$
w_{(i)}(r, t)=\sum_{n=0}^{\infty} w_{(i) n}(r, t), \quad i=1,2 .
$$

To find the components $w_{(i) 0}, w_{(i) 1}, w_{(i) 2}, \ldots$, separately, decomposition method is used. 
Consider the following nonlinear differential equation:

$$
\begin{gathered}
L_{t} w_{(i)}(r, t)+L_{r} w_{(i)}(r, t)+R_{(i)} w_{(i)}(r, t) \\
+N_{(i)} w_{(i)}(r, t)=g_{(i)}(r, t), \\
L_{r} w_{(j)}(r, t)=g_{(j)}(r, t)-L_{t} w_{(j)}(r, t) \\
-R_{(j)} w_{(j)}(r, t)-N_{(j)} w_{(j)}(r, t) .
\end{gathered}
$$

Here $L_{r}=\partial^{2} / \partial r^{2}$ and $L_{t}=\partial / \partial t$ are linear operators, $g_{(i)}(r, t)$ is a source term, $R_{(i)} w_{(i)}(r, t)$ is a remainder linear operator, and $N_{(i)} u_{(i)}(r, t)$ is a nonlinear term.

Applying $L_{r}^{-1}$ on (A.3) to both side we have

$$
\begin{aligned}
L_{r}^{-1} L_{r} w_{(i)}(r, t)= & L_{r}^{-1} g_{(i)}(r, t)-L_{r}^{-1} L_{t} w_{(i)}(r, t) \\
& -L_{r}^{-1} R_{(i)} w_{(i)}(r, t) \\
& -L_{r}^{-1} N_{(i)} w_{(i)}(r, t), \\
w_{(i)}(r, t)= & f_{(i)}(r, t)-L_{r}^{-1} L_{t} w_{(i)}(r, t) \\
& -L_{r}^{-1} R_{(i)} w_{(i)}(r, t) \\
& -L_{r}^{-1} N_{(i)} w_{(i)}(r, t) .
\end{aligned}
$$

The function $f_{(j)}(r, t)$ is obtained by utilizing the boundary conditions given in (20) and (21). The operator $L_{r}^{-1}=$ $\iint(\cdot) d r d r$ is used for second-order differential equations.

With the series solution of $w_{(i)}$ using ADM, we have

$$
\begin{aligned}
w_{(i)}(r, t)= & \sum_{n=0}^{\infty} w_{(i) n}(r, t), \\
\sum_{n=0}^{\infty} w_{(i) n}(r, t)= & f_{(i)}(r, t)-L_{r}^{-1} R_{(i)} \sum_{n=0}^{\infty} w_{(i) n}(r, t) \\
& -L_{r}^{-1} N_{(i)} \sum_{n=0}^{\infty} w_{(i) n}(r, t)
\end{aligned}
$$

In view of Adomian polynomials the nonlinear term $N_{(i)} \sum_{n=0}^{\infty} w_{(i) n}(r, t)$ can be expressed as

$$
N_{(i)} \sum_{n=0}^{\infty} w_{(i) n}(r, t)=\sum_{n=0}^{\infty} \mathbf{A}_{(i) n},
$$

where the components $w_{(i) 0}, w_{(i) 1}, w_{(i) 2}, w_{(i) 3}, \ldots$ are determined as

$$
\begin{aligned}
w_{(i) 0} & +w_{(i) 1}+w_{(i) 2}+w_{(i) 3}+w_{(i) 4}+\cdots \\
= & f_{(i)}(r, t) \\
& -L_{r}^{-1} R_{(i)}\left(w_{(i) 0}+w_{(i) 1}+w_{(i) 2}+w_{(i) 3}+\cdots\right) \\
& -L_{r}^{-1} N_{(i)}\left(\mathbf{A}_{(i) 0}+\mathbf{A}_{(i) 1}+\cdots\right) .
\end{aligned}
$$

To determine the series components $w_{(i) 0}, w_{(i) 1}, w_{(i) 2}$, $w_{(i) 3}, \ldots$, it should be noted that $\mathrm{ADM}$ suggest that $f_{(j)}(r, t)$, in fact describe the zeroth component $w_{(i) 0}$.
The recursive relation is defined as

$$
\begin{aligned}
& w_{(i) 0}(r, t)=f_{(i) 0}(r, t) \\
& w_{(i) 1}(r, t)=-L_{r}^{-1} R_{(i)}\left[w_{(i) 0}(r, t)\right]-L_{r}^{-1}\left(\mathbf{A}_{(i) 0}\right), \\
& w_{(i) 2}(r, t)=-L_{r}^{-1} R_{(i)}\left[w_{(i) 1}(r, t)\right]-L_{r}^{-1}\left(\mathbf{A}_{(i) 1}\right), \\
& w_{(i) 3}(r, t)=-L_{r}^{-1} R_{(i)}\left[w_{2}(r, t)\right]-L_{r}^{-1}\left(\mathbf{A}_{(i) 2}\right),
\end{aligned}
$$

and so on.

\section{B. Analysis of OHAM}

To study the basic idea of OHAM, we consider the following nonlinear differential equation:

$$
\begin{aligned}
A\left(w_{(i)}(r)\right)+G_{(i)}(r) & =0, \quad r \in \Lambda, \\
B\left(w_{(i)}, \frac{d w_{(i)}}{d r}\right) & =0, \quad r \in \Delta,
\end{aligned}
$$

where $A$ is the differential operator and $B$ is the boundary operator, $w_{(i)}$ is the unknown function, $r$ is the spatial independent variable, $\Delta$ is the boundary of the domain $\Lambda$, and $G_{(i)}(r)$ is the unknown analytical function. The operator $A$ can be written as

$$
A=L_{(i)}+N_{(i)}
$$

where $L_{(i)}$ and $N_{(i)}$ are the linear and nonlinear operators, respectively.

From (9), we have

$$
\begin{aligned}
& L_{(j)}\left[\varphi_{(j)}(r, p)\right]=r \frac{d^{2} w_{(j)}}{d r^{2}}+\frac{d w_{(j)}}{d r}, \\
& N_{(j)}\left[\varphi_{(j)}(r, p)\right] \\
& \quad=2 \beta_{(j)}\left(3 r \frac{d^{2} w_{(j)}}{d r^{2}}\left(\frac{d w_{(j)}}{d r}\right)^{2}+\left(\frac{d w_{(j)}}{d r}\right)^{3}\right), \\
& G_{(j)}=0,
\end{aligned}
$$

where $L_{(j)}, N_{(j)}$, and $G_{(j)}$ are linear operator, nonlinear operator, and source term, respectively, and $p \in[0,1]$ is an embedding parameter.

We consider a homotopy $\varphi_{(j)}(r, p): \Lambda \times[0,1] \rightarrow R$ that satisfies

$$
\begin{aligned}
& {[1-p]\left[L_{(j)}\left[\varphi_{(j)}(r, p)\right]+G_{(j)}(r)\right]-H_{(j)}(p)} \\
& \quad \cdot\left[L_{(j)}\left[\varphi_{(j)}(r, p)\right]+N_{(j)}\left[\varphi_{(j)}(r, p)\right]+G_{j}(r)\right] \\
& \quad=0,
\end{aligned}
$$

$$
B\left(\varphi_{(j)}(r, p), \frac{\partial \varphi_{(j)}(r, p)}{\partial r}\right)=0,
$$


with boundary conditions

$$
\begin{aligned}
\varphi_{1}(1, p) & =1, \\
\varphi_{2}(\delta, p) & =U, \\
\varphi_{1}(\Omega, p) & =\varphi_{2}(\Omega, p), \\
S_{r z(1)}(\Omega, p) & =S_{r z(2)}(\Omega, p),
\end{aligned}
$$

where $H_{(j)}(p)$ is a nonzero auxiliary function and $\varphi_{(j)}(r, p)$ is an unknown function. For $p=0$, (11) only recuperate the linear part of solution; that is, $\varphi_{(j)}(r, p)=w_{(j) 0}(r)$,

$$
\begin{gathered}
L_{(j)}\left[\varphi_{(j)}(r, 0)\right]+G(r)=0, \\
B\left(w_{(j) 0}, \frac{\partial w_{(j) 0}}{\partial r}\right),
\end{gathered}
$$

For $p=1$, we recuperate the nonlinear boundary value problem and the solution converges to exact solution; that is, $\varphi_{(j)}(r, 1)=w_{(j)}(r)$. The solution $\varphi_{(j)}(r, p)$ approaches from $w_{(j) 0}$ to $w_{(j)}(r)$ as $p$ varies from 0 to 1 .

In order to improve the accuracy of the results and also in order to ensure a faster convergence to the exact solution, we use the following generalized auxiliary function involving an increased number of convergence-control parameters even in the first order of approximation, including also a physical parameter or a function of the physical parameter

$$
H\left(t ; p, C_{i}\right)=p H_{1}\left(t, C_{i}\right)+p^{2} H_{2}\left(t, C_{i}\right)+\cdots,
$$

where $H_{i}\left(t ; C_{j}\right), i=1,2, \ldots$, are auxiliary functions depending on it (or another physical parameter) and unknown convergence-control parameters $C_{j}, j=1,2, \ldots$.

For approximate solution, $\varphi_{(j)}(r, p)$ is expanding with respect to $p$ by using Taylor series

$$
\begin{array}{r}
\varphi_{(j)}\left(r, p, C_{i}\right)=w_{(j) 0}(r)+\sum_{k=1}^{\infty} w_{(j) k}\left(r, C_{i}\right) p^{k}, \\
i=1,2, \ldots .
\end{array}
$$

Substituting both (B.7) and (B.8) into (B.4), and equating each coefficient like power of $p$ and $\bar{p}$, we have different order problems.

Zeroth-order problem with boundary conditions is shown as follows:

$$
\begin{aligned}
p^{0}: r \frac{d^{2} w_{(1) 0}}{d r^{2}}+\frac{d w_{(1) 0}}{d r} & =0, \\
p^{0}: r \frac{d^{2} w_{(2) 0}}{d r^{2}}+\frac{d w_{(2) 0}}{d r} & =0, \\
w_{(1) 0}(1) & =1, \\
w_{(2) 0}(\delta) & =U, \\
w_{(1) 0}(\Omega) & =w_{(2) 0}(\Omega), \\
S_{r z(1) 0}(\Omega) & =S_{r z(2) 0}(\Omega) .
\end{aligned}
$$

First-order problem with boundary conditions is shown as follows:

$$
\begin{aligned}
& p^{1}: r \frac{d^{2} w_{(1) 1}}{d r^{2}}+\frac{d w_{(1) 1}}{d r}-\frac{d w_{(1) 0}}{d r}-C_{1} \frac{d w_{(1) 0}}{d r} \\
& -2 \beta_{1} C_{1}\left(\frac{d w_{(1) 0}}{d r}\right)^{3}-r \frac{d^{2} w_{(1) 0}}{d r^{2}}-r C_{1} \frac{d^{2} w_{(1) 0}}{d r^{2}} \\
& -6 r \beta_{1} C_{1} \frac{d^{2} w_{(1) 0}}{d r^{2}}\left(\frac{d w_{(1) 0}}{d r}\right)^{2}=0, \\
& p^{1}: \frac{d^{2} w_{(2) 1}}{d r^{2}}+\frac{d w_{(2) 1}}{d r}-\frac{d w_{(1) 0}}{d r}-C_{3} \frac{d w_{(2) 0}}{d r} \\
& -2 \beta_{2} C_{3}\left(\frac{d w_{(2) 0}}{d r}\right)^{3}-r \frac{d^{2} w_{(2) 0}}{d r^{2}}-r C_{3} \frac{d^{2} w_{(2) 0}}{d r^{2}} \\
& -6 r \beta_{2} C_{3} \frac{d^{2} w_{(2) 0}}{d r^{2}}\left(\frac{d w_{(2) 0}}{d r}\right)^{2}=0, \\
& w_{(1) 1}(1)=0, \\
& w_{(2) 1}(\delta)=0, \\
& w_{(1) 1}(\Omega)=w_{(2) 1}(\Omega), \\
& S_{r z(1) 1}(\Omega)=S_{r z(2) 1}(\Omega) .
\end{aligned}
$$

Second-order problem with boundary conditions is shown as follows:

$$
\begin{aligned}
p^{2} & : r \frac{d^{2} w_{(1) 2}}{d r^{2}}+\frac{d w_{(1) 2}}{d r}-C_{2} \frac{d w_{(1) 0}}{d r} \\
& -2 \beta_{1} C_{2}\left(\frac{d w_{(1) 0}}{d r}\right)^{3}-\frac{d w_{(1) 1}}{d r}-r \frac{d^{2} w_{(1) 0}}{d r^{2}} \\
& -r C_{2} \frac{d^{2} w_{(1) 0}}{d r^{2}}-C_{1} \frac{d w_{(1) 1}}{d r} \\
& -6 r \beta_{1} C_{1} \frac{d w_{(1) 1}}{d r}\left(\frac{d w_{(1) 0}}{d r}\right)^{2} \\
& -6 r \beta_{1} C_{2} \frac{d^{2} w_{(1) 0}}{d r^{2}}\left(\frac{d w_{(1) 0}}{d r}\right)^{2} \\
& -12 r \beta_{1} C_{1} \frac{d^{2} w_{(1) 0}}{d r^{2}} \frac{d w_{(1) 0}}{d r} \frac{d w_{(1) 1}}{d r}-r \frac{d^{2} w_{(1) 1}}{d r^{2}} \\
& -r C_{1} \frac{d^{2} w_{(1) 1}}{d r^{2}}-6 r \beta_{1} C_{1} \frac{d^{2} w_{(1) 1}}{d r^{2}}\left(\frac{d w_{(1) 0}}{d r}\right)^{2}=0, \\
\dot{p}^{2}: & r \frac{d^{2} w_{(2) 2}}{d r^{2}}+\frac{d w_{(2) 2}}{d r}-C_{4} \frac{d w_{(2) 0}}{d r}
\end{aligned}
$$




$$
\begin{aligned}
& -2 \beta_{2} C_{4}\left(\frac{d w_{(2) 0}}{d r}\right)^{3}-\frac{d w_{(2) 1}}{d r}-r \frac{d^{2} w_{(2) 0}}{d r^{2}} \\
& -r C_{4} \frac{d^{2} w_{(2) 0}}{d r^{2}}-C_{3} \frac{d w_{(2) 1}}{d r} \\
& -6 r \beta_{2} C_{3} \frac{d w_{(2) 1}}{d r}\left(\frac{d w_{(2) 0}}{d r}\right)^{2} \\
& -6 r \beta_{2} C_{4} \frac{d^{2} w_{(2) 0}}{d r^{2}}\left(\frac{d w_{(2) 0}}{d r}\right)^{2} \\
& -12 r \beta_{2} C_{3} \frac{d^{2} w_{(2) 0}}{d r^{2}} \frac{d w_{(2) 0}}{d r} \frac{d w_{(2) 1}}{d r}-r \frac{d^{2} w_{(2) 1}}{d r^{2}} \\
& -r C_{3} \frac{d^{2} w_{(2) 1}}{d r^{2}}-6 r \beta_{2} C_{3} \frac{d^{2} w_{(2) 1}}{d r^{2}}\left(\frac{d w_{(2) 0}}{d r}\right)^{2}=0, \\
& w_{(1) 2}(1)=0, \\
& w_{(2) 2}(\delta)=0, \\
& w_{(1) 2}(\Omega)=w_{(2) 2}(\Omega), \\
& S_{r z(1) 2}(\Omega)=S_{r z(1) 2}(\Omega) .
\end{aligned}
$$

The convergence of (B.8) depends upon the auxiliary constant and order of the problem.
If it converges at $p=1$, one has

$$
\begin{aligned}
& w_{(j)}\left(r, C_{1}, C_{2}, \ldots, C_{n}\right) \\
& \quad=w_{(j) 0}(r)+\sum_{n=1}^{\infty} w_{(j) n}\left(r, C_{1}, C_{2}, \ldots, C_{n}\right) .
\end{aligned}
$$

Using (B.12) in (B.1), the expression for the residual in the following is obtained as

$$
\begin{aligned}
R_{(j)}\left(r, C_{n}\right)= & L_{(j)} w\left(r, C_{n}\right)+G_{(j)}(r) \\
& +N_{(j)}\left(w\left(r, C_{n}\right)\right), \quad n=1,2, \ldots, m .
\end{aligned}
$$

Many methods such as Ritz Method, Galerkin's Method, collocation method, and least square method are used to find the auxiliary constants.

Here we use the least square method to find the auxiliary constant:

$$
\begin{aligned}
J\left(C_{1}, C_{2}, \ldots, C_{m}\right) & =\int_{a}^{b} R_{(j)}{ }^{2}\left(r, C_{1}, C_{2}, \ldots, C_{m}\right) d r, \\
\frac{\partial J}{\partial C_{1}} & =\frac{\partial J}{\partial C_{2}}=\cdots=0,
\end{aligned}
$$

where $a$ and $b$ (taking from domain) are constant that locate the auxiliary constants which minimize the residual.

The above sequence of problems given in (B.9)-(B.11) are solved using Mathematica software (see (26) in Solution of the Problem):

$$
\begin{aligned}
\sigma_{1} & =1 \\
\sigma_{3} & =U-\sigma_{4} \ln \delta \\
\sigma_{2} & =\frac{U-1}{\ln \Omega}-\sigma_{4}\left(\frac{\ln \delta}{\ln \Omega}-1\right), \\
\sigma_{4} & =-\frac{p_{2}}{3 p_{3}}-\frac{2^{1 / 3}\left(-p_{2}^{2}+3 p_{1} p_{3}\right)}{3 p_{3}\left(-2 p_{2}^{3}+9 p_{1} p_{2} p_{3}-27 p_{3}^{2} p_{4}+\sqrt{4\left(-p_{2}^{2}+3 p_{1} p_{3}\right)^{3}+\left(-2 p_{2}^{3}+9 p_{1} p_{2} p_{3}-27 p_{3}^{2} p_{4}\right)^{2}}\right)^{1 / 3}} \\
& +\frac{\left(-2 p_{2}^{3}+9 p_{1} p_{2} p_{3}-27 p_{3}^{2} p_{4}+\sqrt{4\left(-p_{2}^{2}+3 p_{1} p_{3}\right)^{3}+\left(-2 p_{2}^{3}+9 p_{1} p_{2} p_{3}-27 p_{3}^{2} p_{4}\right)^{2}}\right)^{1 / 3}}{32^{1 / 3} p_{3}}, \\
p_{1} & =1+\left(\frac{\ln \delta}{\ln \Omega}-1\right)\left(1-\frac{6 \gamma_{1}}{\Omega^{2}}\left(\frac{U-1}{\ln \Omega}\right)^{2}\right), \\
p_{2} & =\frac{6 \gamma_{1}}{\Omega^{2}}\left(\frac{U-1}{\ln \Omega}\right)\left(\frac{\ln \delta}{\ln \Omega}-1\right)^{2}, \\
p_{3}= & -\frac{2}{\Omega^{2}}\left(\gamma_{2}-\gamma_{1}\left(\frac{\ln \delta}{\ln \Omega}-1\right)^{3}\right), \\
p_{4} & =\frac{U-1}{\ln \Omega},
\end{aligned}
$$




$$
\begin{aligned}
& \sigma_{5}=k_{2}-\frac{\beta_{1} C_{1} k_{2}^{2}}{8}+\frac{\beta_{1} C_{2} k_{2}^{2}}{2} \\
& \sigma_{6}=\frac{1}{\ln \Omega} \Omega k_{2}+C_{1} k_{2}^{2} \beta_{1}+\frac{\lambda C_{1} k_{2}^{2} \beta_{1}}{2 \Omega^{2}}+C_{3} k_{2} \ln \delta-\Omega k_{2}-C_{3} k_{2} \ln \Omega-\sigma_{8}(\ln (\Omega \delta)), \\
& \sigma_{7}=-\sigma_{8} \ln \delta+\delta k_{2}+C_{3} k_{2} \ln \delta-\frac{\lambda C_{4} k_{2}^{2} \beta_{2}}{8 \delta^{4}}+\frac{\lambda C_{3} k_{2}^{2} \beta_{2}}{2 \delta^{2}}, \\
& \sigma_{8}=\frac{\lambda}{(\zeta / \Omega+\ln (\delta \Omega))}\left[C_{1} k_{2} \ln \Omega-C_{1} k_{2}^{2} \beta_{0}+\frac{C_{1} k_{2}^{2} \beta_{1}}{2 \Omega^{2}}+C_{3} k_{2} \ln \delta-\frac{C_{4} k_{2}^{2} \beta_{2}}{8 \delta^{4}}+\frac{C_{3} k_{2}^{2} \beta_{2}}{2 \delta^{2}}-C_{3} k_{2} \ln \Omega+\frac{C_{4} k_{2}^{2} \beta_{2}}{8 \Omega^{4}}\right] \\
& -\frac{C_{3} k_{2}^{2} \beta_{2}}{2 \Omega^{2}}-\frac{\Omega \ln \Omega}{(\zeta / \Omega+\ln (\delta \Omega))}\left[k_{2}+\frac{C_{1} k_{2}}{\Omega}+\frac{C_{1} k_{2}^{2} \beta_{1}}{2 \Omega^{5}}-\frac{C_{1} k_{2}^{2} \beta_{1}}{\Omega^{3}}-\frac{C_{1} k_{2}^{2} \beta_{1}}{\Omega^{3}}\right. \\
& \left.-\zeta\left(k_{2}+\frac{C_{3} k_{2}}{\Omega}+\frac{C_{1} k_{2}^{2} \beta_{1}}{2 \Omega^{5}}-\frac{C_{3} k_{2}^{2} \beta_{1}}{\Omega^{3}}\right)\right] \text {, } \\
& \sigma_{9}=-k_{2}\left(1+C_{1}^{2}\right)+\frac{16132}{48} \lambda C_{1} k_{2}^{2} \beta_{1}+\frac{83}{240} C_{1}^{2} k_{2}^{2} \beta_{1}+C_{2} k_{2}^{2} \beta_{1}-3 C_{1}^{2} k_{2}^{2} \beta_{1}-6 C_{1} k_{2}^{3} \beta_{1}-\frac{3}{2} C_{1}^{2} k_{2}^{3} \beta_{1}+\frac{5}{6} C_{1}^{2} k_{2}^{4} \beta_{1}^{2}+\sigma_{4}(1 \\
& \left.+k_{1}+3 C_{1} k_{2}^{2} \beta_{1}\right) \text {, } \\
& \sigma_{10}=\frac{\lambda}{\ln \Omega}\left[-\sigma_{9}-2 k_{2}\left(C_{1}\left(1+C_{1}\right)-C_{3}\left(1+C_{3}\right)-\frac{3}{2}\left(\sigma_{4}+\sigma_{6}\right)\right) \ln \Omega-\frac{k_{2}}{\Omega}\left(C_{1}^{3}-C_{3}^{3}+C_{1}^{2}-C_{3}^{2}\right)+\Omega\left(C_{1}+C_{3}\right)\right. \\
& +\left(\frac{k_{2}^{2}}{10 \Omega^{5}}-\frac{k_{2}^{2}}{32 \Omega^{4}}\right)\left(\beta_{1} C_{1}+\beta_{2} C_{3}\right)+\left(\frac{k_{2}^{2}}{3 \Omega^{3}}+\frac{k_{2}^{2}}{4 \Omega^{2}}\right)\left(\beta_{1} C_{1}+\beta_{2} C_{3}\right)+\left(\frac{k_{2}^{2}}{10 \Omega^{5}}+\frac{k_{2}^{2}}{32 \Omega^{4}}\right)\left(\beta_{1} C_{1}^{2}+\beta_{2} C_{3}^{2}\right) \\
& +\frac{k_{2}^{2}}{3 \Omega^{3}}\left(\beta_{1} C_{1}^{2}+\beta_{2} C_{3}^{2}\right)-\frac{k_{2}^{2}}{4 \Omega^{4}}\left(\left(\beta_{1} C_{1}^{2}-\beta_{2} C_{3}^{2}\right)+\left(\beta_{1} C_{2}+\beta_{2} C_{4}\right)\right)-\left(\frac{3 k_{1} k_{2}^{2}}{\Omega^{2}}-\frac{3 k_{2}^{2}}{2 \Omega^{2}}\right)\left(\beta_{1} C_{1}^{2}-\beta_{2} C_{3}^{2}\right) \\
& \left.+\frac{k_{2}^{4}}{3 \Omega^{6}}\left(\beta_{1} C_{1}^{2}+\beta_{2} C_{3}^{2}\right)-\frac{k_{2}^{2}}{4 \Omega^{2}}\left(\beta_{1} C_{1}-\beta_{2} C_{3}\right)\right]+\frac{k_{2}^{4}}{2 \Omega^{4}}\left(\beta_{1} C_{1}^{2}+\beta_{2} C_{3}^{2}\right)+\frac{1}{\Omega}\left(\sigma_{4}+\sigma_{6}\right)+\frac{1}{\Omega}\left(C_{1} \sigma_{4}+C_{3} \sigma_{6}\right) \\
& +\frac{3 k_{2}^{4}}{\Omega^{2}}\left(\beta_{1} C_{1} \sigma_{4}+\beta_{2} C_{3} \sigma_{6}\right)+\sigma_{11}+\sigma_{12} \ln \Omega \\
& \sigma_{11}=-\left[\sigma_{12} \ln \Omega+\lambda 2 k_{1}\left(C_{3}-C_{3}^{2}\right) \ln \Omega-\delta k_{2}+\frac{\lambda k_{2}}{\delta} C_{3}-\delta k_{2} C_{3}+\frac{k_{2}}{\delta} C_{3}^{2}-\frac{k_{2}^{2}}{10 \delta^{5}} \beta_{2} C_{3}+\frac{\lambda k_{2}^{2}}{32 \Omega^{4}} \beta_{2} C_{3}-\frac{k_{2}^{2}}{3 \delta^{3}} \beta_{2} C_{3}\right. \\
& -\frac{k_{2}^{2}}{10 \delta^{5}} \beta_{2} C_{3}^{2}-\frac{k_{2}^{2}}{32 \delta^{4}} \beta_{2} C_{3}^{2}+\frac{k_{2}^{2}}{3 \delta^{3}} \beta_{2} C_{3}^{2}+\frac{k_{2}^{2}}{4 \delta^{2}} \beta_{2} C_{3}^{2}-\frac{k_{2}^{2}}{\delta^{2}} \beta_{2} C_{4}+\frac{3 k_{1} k_{2}^{2}}{\delta^{2}} \beta_{2} C_{3}^{2}+\frac{6 k_{2}^{3}}{\delta} \beta_{2} C_{3}+\frac{3 k_{2}^{3}}{2 \delta^{3}} \beta_{2} C_{3}^{2}+\frac{k_{2}^{4}}{3 \delta^{6}} \beta_{2} C_{3}^{2} \\
& \left.-\frac{\sigma_{4}}{\delta}-\sigma_{4} \ln \delta+C_{1} \sigma_{4} \ln \delta-\frac{3 k_{2}^{2}}{\delta^{2}} \beta_{2} C_{3}^{2} \sigma_{4}\right] \text {, } \\
& \sigma_{12}=\left(\frac{\lambda}{\Omega}(\ln \delta-1)\right)\left[-2 k_{1}\left(C_{1}-C_{3}\right) \ln \Omega-2 k_{1}\left(C_{1}^{3}-C_{3}^{3}\right) \ln \Omega+\frac{k_{2}}{\Omega^{2}}\left(C_{1}+C_{3}\right)-k_{2}\left(C_{1}-C_{3}\right)+\frac{k_{2}}{\Omega^{2}}\left(C_{1}^{2}+C_{3}^{2}\right)\right. \\
& +\frac{k_{2}^{2}}{2 \Omega^{6}}\left(\beta_{1} C_{1}+\beta_{2} C_{3}\right)+\frac{k_{2}^{2}}{8 \Omega^{5}}\left(\beta_{1} C_{1}+\beta_{2} C_{3}\right)+\frac{k_{2}^{2}}{\Omega^{4}}\left(\beta_{1} C_{1}+\beta_{2} C_{3}\right)+\frac{k_{2}^{2}}{2 \Omega^{3}}\left(\beta_{1} C_{1}+\beta_{2} C_{3}\right)+\frac{k_{2}^{2}}{2 \Omega^{6}}\left(\beta_{1} C_{1}^{2}+\beta_{2} C_{3}^{2}\right) \\
& +\frac{k_{2}^{2}}{8 \Omega^{5}}\left(\beta_{1} C_{1}^{2}+\beta_{2} C_{3}^{2}\right)+\frac{k_{2}^{2}}{\Omega^{4}}\left(\beta_{1} C_{1}^{2}+\beta_{2} C_{3}^{2}\right)+\frac{k_{2}^{2}}{2 \Omega^{3}}\left(\beta_{1} C_{1}^{2}+\beta_{2} C_{3}^{2}\right)+\frac{2 k_{2}^{2}}{\Omega^{3}}\left(\beta_{1} C_{2}^{2}+\beta_{2} C_{4}^{2}\right)+\frac{3 k_{1} k_{2}^{2}}{\Omega^{3}}\left(\beta_{1} C_{1}^{2}+\beta_{2} C_{3}^{2}\right) \\
& +\frac{6 k_{2}^{2}}{\Omega^{2}}\left(\beta_{1} C_{1}+\beta_{2} C_{3}\right)+\frac{3 k_{2}^{3}}{\Omega^{3}}\left(\beta_{1} C_{1}^{2}+\beta_{2} C_{3}^{2}\right)-\frac{2 k_{2}^{4}}{\Omega^{7}}\left(\beta_{1} C_{1}^{2}+\beta_{2} C_{3}^{2}\right)-\frac{6 k_{2}^{4}}{\Omega^{5}}\left(\beta_{1}^{2} C_{1}^{2}-\beta_{2}^{2} C_{3}^{2}\right)-\frac{1}{\Omega^{2}}\left(\sigma_{4}-\sigma_{6}\right)
\end{aligned}
$$




$$
\begin{aligned}
& \left.+\left(\sigma_{4}+\sigma_{6}\right) \ln \Omega-\frac{1}{\Omega^{2}}\left(C_{1} \sigma_{4}-C_{3} \sigma_{6}\right)-\left(C_{1} \sigma_{4}-C_{3} \sigma_{6}\right) \ln \Omega-\frac{6 k_{2}^{2}}{\Omega^{3}}\left(\beta_{1} C_{1} \sigma_{4}-\beta_{2} C_{3} \sigma_{6}\right)+\frac{1}{\ln \Omega} \sigma_{9}\right], \\
& \Phi_{11}=1-\beta_{1}^{2} C_{1} k_{2}+\beta_{1} \sigma_{5} \\
& \Gamma_{11}=1-\beta_{2}^{2} C_{3} k_{2}+\beta_{2} \sigma_{7} \text {, } \\
& \Phi_{12}=\frac{U-1}{\ln \delta}+\beta_{1} \sigma_{6}-\beta_{1} C_{1} k_{2}+\beta_{1}^{2} \sigma_{10}, \\
& \Gamma_{12}=\frac{U-1}{\ln \delta}+\beta_{2} \sigma_{8}-\beta_{2} C_{3} k_{2}+\beta_{2}^{2} \sigma_{12} \\
& \Phi_{13}=\beta_{1} k_{2}-\beta_{1}^{2} k_{2} \text {, } \\
& \Gamma_{13}=\beta_{2} k_{2}-\beta_{2}^{2} k_{2} \text {, } \\
& \Phi_{14}=\beta_{1}^{2} C_{1}^{2} k_{2}+6 \beta_{1} C_{1} k_{2}^{3}-\sigma_{4}\left(1+C_{1}\right)+\beta_{1}^{2} C_{1} k_{2}, \\
& \Gamma_{14}=\beta_{2}^{2} C_{3}^{2} k_{2}+6 \beta_{2} C_{3} k_{2}^{3}-\sigma_{6}\left(1+C_{3}\right)+\beta_{2}^{2} C_{3} k_{2} \text {, } \\
& \Phi_{15}=-\frac{1}{2} C_{1} k_{2}^{2} \beta_{1}^{2}-\frac{1}{4} C_{1} k_{2}^{2} \beta_{1}^{3}+3 C_{1} k_{2}^{2} \beta_{1}^{2} \sigma_{4}-\frac{1}{4} C_{1} k_{2}^{2} \beta_{1}^{2}+\frac{1}{2} C_{1}^{2} k_{2}^{2} \beta_{1}^{2}+3 C_{1}^{2} k_{2}^{2} \beta_{1}^{3} k_{1}-C_{2}^{2} k_{2}^{2} \beta_{1}^{3} \text {, } \\
& \Phi_{16}=-\frac{1}{3} C_{1} k_{2}^{2} \beta_{1}^{3}-\frac{1}{3} C_{1}^{2} k_{2}^{2} \beta_{1}^{3} \text {, } \\
& \Gamma_{16}=-\frac{1}{3} C_{3} k_{2}^{2} \beta_{2}^{3}-\frac{1}{3} C_{3}^{2} k_{2}^{2} \beta_{2}^{3} \text {, } \\
& \Phi_{17}=\frac{1}{8} C_{1} k_{2}^{2} \beta_{1}^{2}-\frac{1}{32} C_{1} k_{2}^{2} \beta_{1}^{3}-\frac{1}{32} C_{1}^{2} k_{2}^{2} \beta_{1}^{3}-\frac{1}{2} C_{1}^{2} k_{2}^{4} \beta_{1}^{3} \text {, } \\
& \Phi_{18}=-\frac{1}{10} C_{1} k_{2}^{2} \beta_{1}^{3}-\frac{1}{10} C_{1}^{2} k_{2}^{2} \beta_{1}^{2}, \\
& \Gamma_{18}=-\frac{1}{10} C_{3} k_{2}^{2} \beta_{2}^{3}-\frac{1}{10} C_{3}^{2} k_{2}^{2} \beta_{2}^{2} \text {, } \\
& \Gamma_{15}=-\frac{1}{2} C_{3} k_{2}^{2} \beta_{2}^{2}-\frac{1}{4} C_{3} k_{2}^{2} \beta_{2}^{3}+3 C_{3} k_{2}^{2} \beta_{2}^{2} \sigma_{6}-\frac{1}{4} C_{3} k_{2}^{2} \beta_{2}^{2}+\frac{1}{2} C_{3}^{2} k_{2}^{2} \beta_{2}^{2}+3 C_{3}^{2} k_{2}^{2} \beta_{2}^{3} k_{1}-C_{4}^{2} k_{2}^{2} \beta_{2}^{3} \text {, } \\
& \Gamma_{17}=\frac{1}{8} C_{3} k_{2}^{2} \beta_{2}^{2}-\frac{1}{32} C_{3} k_{2}^{2} \beta_{2}^{3}-\frac{1}{32} C_{3}^{2} k_{2}^{2} \beta_{2}^{3}-\frac{1}{2} C_{3}^{2} k_{2}^{4} \beta_{2}^{3} \text {, } \\
& \Psi_{1}=-\frac{625}{242} B r_{1} \beta_{1} \Phi_{18}^{4} \\
& \Psi_{2}=-\frac{4000}{441} B r_{1} \beta_{1} \Phi_{17} \Phi_{18}^{3} \\
& \Psi_{3}=-12 B r_{1} \beta_{1} \Phi_{17}^{2} \Phi_{18}^{2}-\frac{15}{2} B r_{1} \beta_{1} \Phi_{16} \Phi_{18}^{3}, \\
& \Psi_{4}=-12 B r_{1} \beta_{1} \Phi_{17}^{2} \Phi_{18}^{2}-\frac{15}{2} B r_{1} \beta_{1} \Phi_{16} \Phi_{18}^{3}, \\
& \Psi_{5}=-\frac{2560}{361} B r_{1} \beta_{1} \Phi_{17}^{3} \Phi_{18}-\frac{7200}{361} B r_{1} \beta_{1} \Phi_{16} \Phi_{17} \Phi_{18}^{2}-\frac{2000}{361} B r_{1} \beta_{1} \Phi_{15} \Phi_{18}^{3} \\
& \Psi_{6}=-\frac{128}{81} B r_{1} \beta_{1} \Phi_{17}^{4}-\frac{160}{9} B r_{1} \beta_{1} \Phi_{16} \Phi_{17}^{2} \Phi_{18}-\frac{25}{3} B r_{1} \beta_{1} \Phi_{16}^{2} \Phi_{18}^{2}-\frac{400}{27} B r_{1} \beta_{1} \Phi_{15} \Phi_{17} \Phi_{18}^{2}-\frac{250}{81} B r_{1} \beta_{1} \Phi_{14} \Phi_{18}^{3}, \\
& \Psi_{8}=-\frac{27}{4} B r_{1} \beta_{1} \Phi_{16}^{2} \Phi_{17}^{2}-4 B r_{1} \beta_{1} \Phi_{15} \Phi_{17}^{3}-\frac{135}{32} B r_{1} \beta_{1} \Phi_{16}^{3} \Phi_{18}-\frac{45}{2} B r_{1} \beta_{1} \Phi_{15} \Phi_{16} \Phi_{17} \Phi_{18}-\frac{15}{2} B r_{1} \beta_{1} \Phi_{14} \Phi_{17}^{2} \Phi_{18}-\frac{75}{16} \\
& \cdot B r_{1} \beta_{1} \Phi_{15}^{2} \Phi_{18}^{2}-\frac{225}{32} B r_{1} \beta_{1} \Phi_{14} \Phi_{16} \Phi_{18}^{2}+\frac{75}{8} B r_{1} \beta_{1} \Phi_{12} \Phi_{17} \Phi_{18}^{2}+\frac{125}{32} B r_{1} \beta_{1} \Phi_{13} \Phi_{18}^{3}
\end{aligned}
$$




$$
\begin{aligned}
& \Psi_{9}=-\frac{96}{25} B r_{1} \beta_{1} \Phi_{16}^{3} \Phi_{17}-\frac{256}{25} B r_{1} \beta_{1} \Phi_{15} \Phi_{16} \Phi_{17}^{2}-\frac{512}{225} B r_{1} \beta_{1} \Phi_{14} \Phi_{17}^{3}-\frac{48}{5} B r_{1} \beta_{1} \Phi_{15} \Phi_{16}^{2} \Phi_{18}-\frac{128}{15} B r_{1} \beta_{1} \Phi_{15}^{2} \Phi_{17} \Phi_{18} \\
& \text { - } \frac{64}{5} B r_{1} \beta_{1} \Phi_{14} \Phi_{16} \Phi_{17} \Phi_{18}+\frac{128}{15} B r_{1} \beta_{1} \Phi_{12} \Phi_{17}^{2} \Phi_{18}-\frac{16}{3} B r_{1} \beta_{1} \Phi_{14} \Phi_{15} \Phi_{18}^{2}+8 B r_{1} \beta_{1} \Phi_{12} \Phi_{16} \Phi_{18}^{2}+\frac{32}{3} \\
& \text { - } B r_{1} \beta_{1} \Phi_{13} \Phi_{17} \Phi_{18}^{2} \\
& \Psi_{10}=-\frac{81}{98} B r_{1} \beta_{1} \Phi_{16}^{4}-\frac{432}{49} B r_{1} \beta_{1} \Phi_{15} \Phi_{16}^{2} \Phi_{17}-\frac{192}{49} B r_{1} \beta_{1} \Phi_{15}^{2} \Phi_{17}^{2}-\frac{288}{49} B r_{1} \beta_{1} \Phi_{14} \Phi_{16} \Phi_{17}^{2}+\frac{128}{49} B r_{1} \beta_{1} \Phi_{12} \Phi_{17}^{3}-\frac{360}{49} \\
& \cdot B r_{1} \beta_{1} \Phi_{15}^{2} \Phi_{16} \Phi_{18}-\frac{270}{49} B r_{1} \beta_{1} \Phi_{14} \Phi_{16}^{2} \Phi_{18}-\frac{480}{49} B r_{1} \beta_{1} \Phi_{14} \Phi_{15} \Phi_{17} \Phi_{18}+\frac{720}{49} B r_{1} \beta_{1} \Phi_{12} \Phi_{16} \Phi_{17} \Phi_{18}+\frac{480}{49} \\
& \cdot B r_{1} \beta_{1} \Phi_{13} \Phi_{17}^{2} \Phi_{18}-\frac{75}{49} B r_{1} \beta_{1} \Phi_{14}^{2} \Phi_{18}^{2}+\frac{300}{49} B r_{1} \beta_{1} \Phi_{12} \Phi_{15} \Phi_{18}^{2}+\frac{450}{49} B r_{1} \beta_{1} \Phi_{13} \Phi_{16} \Phi_{18}^{2} \text {, } \\
& \Psi_{11}=-\frac{432}{169} B r_{1} \beta_{1} \Phi_{15} \Phi_{16}^{3}-\frac{1152}{169} B r_{1} \beta_{1} \Phi_{15}^{2} \Phi_{16} \Phi_{17}-\frac{864}{169} B r_{1} \beta_{1} \Phi_{14} \Phi_{16}^{2} \Phi_{17}-\frac{768}{169} B r_{1} \beta_{1} \Phi_{14} \Phi_{15} \Phi_{17}^{2}+\frac{1152}{169} \\
& \cdot B r_{1} \beta_{1} \Phi_{12} \Phi_{16} \Phi_{17}^{2}+\frac{512}{169} B r_{1} \beta_{1} \Phi_{13} \Phi_{17}^{3}-\frac{320}{169} B r_{1} \beta_{1} \Phi_{15}^{3} \Phi_{18}-\frac{1440}{169} B r_{1} \beta_{1} \Phi_{14} \Phi_{15} \Phi_{16} \Phi_{18}+\frac{1080}{169} B r_{1} \beta_{1} \Phi_{12} \Phi_{16}^{2} \Phi_{18} \\
& -\frac{480}{169} B r_{1} \beta_{1} \Phi_{14}^{2} \Phi_{17} \Phi_{18}+\frac{1920}{169} B r_{1} \beta_{1} \Phi_{12} \Phi_{15} \Phi_{17} \Phi_{18}+\frac{2880}{169} B r_{1} \beta_{1} \Phi_{13} \Phi_{16} \Phi_{17} \Phi_{18}+\frac{600}{169} B r_{1} \beta_{1} \Phi_{12} \Phi_{14} \Phi_{18}^{2}+\frac{1200}{169} \\
& \text { - } B r_{1} \beta_{1} \Phi_{13} \Phi_{15} \Phi_{18}^{2} \\
& \Psi_{12}=-3 B r_{1} \beta_{1} \Phi_{15}^{2} \Phi_{16}^{2}-\frac{3}{2} B r_{1} \beta_{1} \Phi_{14} \Phi_{16}^{3}-\frac{16}{9} B r_{1} \beta_{1} \Phi_{15}^{3} \Phi_{17}-8 B r_{1} \beta_{1} \Phi_{14} \Phi_{15} \Phi_{16} \Phi_{17}+6 B r_{1} \beta_{1} \Phi_{12} \Phi_{16}^{2} \Phi_{17}-\frac{4}{3} \\
& \cdot B r_{1} \beta_{1} \Phi_{14}^{2} \Phi_{17}^{2}+\frac{16}{3} B r_{1} \beta_{1} \Phi_{12} \Phi_{15} \Phi_{17}^{2}+8 B r_{1} \beta_{1} \Phi_{13} \Phi_{16} \Phi_{17}^{2}-\frac{10}{3} B r_{1} \beta_{1} \Phi_{14} \Phi_{15}^{2} \Phi_{18}-\frac{5}{2} B r_{1} \beta_{1} \Phi_{14}^{2} \Phi_{16} \Phi_{18} \\
& +10 B r_{1} \beta_{1} \Phi_{12} \Phi_{15} \Phi_{16} \Phi_{18}+\frac{15}{2} B r_{1} \beta_{1} \Phi_{13} \Phi_{16}^{2} \Phi_{18}+\frac{20}{3} B r_{1} \beta_{1} \Phi_{12} \Phi_{14} \Phi_{17} \Phi_{18}+\frac{40}{3} B r_{1} \beta_{1} \Phi_{13} \Phi_{15} \Phi_{17} \Phi_{18}-\frac{25}{12} \\
& \cdot B r_{1} \beta_{1} \Phi_{12}^{2} \Phi_{18}^{2}+\frac{25}{6} B r_{1} \beta_{1} \Phi_{13} \Phi_{14} \Phi_{18}^{2} \\
& \Psi_{13}=-\frac{192}{121} B r_{1} \beta_{1} \Phi_{15}^{3} \Phi_{16}-\frac{432}{121} B r_{1} \beta_{1} \Phi_{14} \Phi_{15} \Phi_{16}^{2}+\frac{216}{121} B r_{1} \beta_{1} \Phi_{12} \Phi_{16}^{3}-\frac{384}{121} B r_{1} \beta_{1} \Phi_{14} \Phi_{15}^{2} \Phi_{17}-\frac{288}{121} \\
& \cdot B r_{1} \beta_{1} \Phi_{14}^{2} \Phi_{16} \Phi_{17}+\frac{1152}{121} B r_{1} \beta_{1} \Phi_{12} \Phi_{15} \Phi_{16} \Phi_{17}+\frac{864}{121} B r_{1} \beta_{1} \Phi_{13} \Phi_{16}^{2} \Phi_{17}+\frac{384}{121} B r_{1} \beta_{1} \Phi_{12} \Phi_{14} \Phi_{17}^{2}+\frac{768}{121} \\
& \cdot B r_{1} \beta_{1} \Phi_{13} \Phi_{15} \Phi_{17}^{2}-\frac{240}{121} B r_{1} \beta_{1} \Phi_{14}^{2} \Phi_{15} \Phi_{18}+\frac{480}{121} B r_{1} \beta_{1} \Phi_{12} \Phi_{15}^{2} \Phi_{18}+\frac{720}{121} B r_{1} \beta_{1} \Phi_{12} \Phi_{14} \Phi_{16} \Phi_{18}+\frac{1440}{121} \\
& \cdot B r_{1} \beta_{1} \Phi_{13} \Phi_{15} \Phi_{16} \Phi_{18}-\frac{480}{121} B r_{1} \beta_{1} \Phi_{12}^{2} \Phi_{17} \Phi_{18}+\frac{960}{121} B r_{1} \beta_{1} \Phi_{13} \Phi_{14} \Phi_{17} \Phi_{18}-\frac{600}{121} B r_{1} \beta_{1} \Phi_{12} \Phi_{13} \Phi_{18}^{2} \\
& \Psi_{14}=-\frac{8}{25} B r_{1} \beta_{1} \Phi_{15}^{4}-\frac{72}{25} B r_{1} \beta_{1} \Phi_{14} \Phi_{15}^{2} \Phi_{16}-\frac{27}{25} B r_{1} \beta_{1} \Phi_{14}^{2} \Phi_{16}^{2}+\frac{108}{25} B r_{1} \beta_{1} \Phi_{12} \Phi_{15} \Phi_{16}^{2}+\frac{54}{25} B r_{1} \beta_{1} \Phi_{13} \Phi_{16}^{3}-\frac{48}{25} \\
& \cdot B r_{1} \beta_{1} \Phi_{14}^{2} \Phi_{15} \Phi_{17}+\frac{96}{25} B r_{1} \beta_{1} \Phi_{12} \Phi_{15}^{2} \Phi_{17}+\frac{144}{25} B r_{1} \beta_{1} \Phi_{12} \Phi_{14} \Phi_{16} \Phi_{17}+\frac{288}{25} B r_{1} \beta_{1} \Phi_{13} \Phi_{15} \Phi_{16} \Phi_{17}-\frac{48}{25} \\
& \cdot B r_{1} \beta_{1} \Phi_{12}^{2} \Phi_{17}^{2}+\frac{96}{25} B r_{1} \beta_{1} \Phi_{13} \Phi_{14} \Phi_{17}^{2}-\frac{2}{5} B r_{1} \beta_{1} \Phi_{14}^{3} \Phi_{18}+\frac{24}{5} B r_{1} \beta_{1} \Phi_{12} \Phi_{14} \Phi_{15} \Phi_{18}+\frac{24}{5} B r_{1} \beta_{1} \Phi_{13} \Phi_{15}^{2} \Phi_{18}-\frac{18}{5} \\
& \text { · } B r_{1} \beta_{1} \Phi_{12}^{2} \Phi_{16} \Phi_{18}+\frac{36}{5} B r_{1} \beta_{1} \Phi_{13} \Phi_{14} \Phi_{16} \Phi_{18}-\frac{48}{5} B r_{1} \beta_{1} \Phi_{12} \Phi_{13} \Phi_{17} \Phi_{18}-\frac{1}{4} B r_{1} \Phi_{18}^{2}-3 B r_{1} \beta_{1} \Phi_{13}^{2} \Phi_{18}^{2} \\
& \Psi_{15}=-\frac{64}{81} B r_{1} \beta_{1} \Phi_{14} \Phi_{15}^{3}-\frac{16}{9} B r_{1} \beta_{1} \Phi_{14}^{2} \Phi_{15} \Phi_{16}+\frac{32}{9} B r_{1} \beta_{1} \Phi_{12} \Phi_{15}^{2} \Phi_{16}+\frac{8}{3} B r_{1} \beta_{1} \Phi_{12} \Phi_{14} \Phi_{16}^{2}+\frac{16}{3} B r_{1} \beta_{1} \Phi_{13} \Phi_{15} \Phi_{16}^{2} \\
& -\frac{32}{81} B r_{1} \beta_{1} \Phi_{14}^{3} \Phi_{17}+\frac{128}{27} B r_{1} \beta_{1} \Phi_{12} \Phi_{14} \Phi_{15} \Phi_{17}+\frac{128}{27} B r_{1} \beta_{1} \Phi_{13} \Phi_{15}^{2} \Phi_{17}-\frac{32}{9} B r_{1} \beta_{1} \Phi_{12}^{2} \Phi_{16} \Phi_{17}+\frac{64}{9}
\end{aligned}
$$




$$
\begin{aligned}
& \cdot B r_{1} \beta_{1} \Phi_{13} \Phi_{14} \Phi_{16} \Phi_{17}-\frac{128}{27} B r_{1} \beta_{1} \Phi_{12} \Phi_{13} \Phi_{17}^{2}+\frac{40}{27} B r_{1} \beta_{1} \Phi_{12} \Phi_{14}^{2} \Phi_{18}-\frac{80}{27} B r_{1} \beta_{1} \Phi_{12}^{2} \Phi_{15} \Phi_{18}+\frac{160}{27} \\
& \cdot B r_{1} \beta_{1} \Phi_{13} \Phi_{14} \Phi_{15} \Phi_{18}-\frac{80}{9} B r_{1} \beta_{1} \Phi_{12} \Phi_{13} \Phi_{16} \Phi_{18}-\frac{40}{81} B r_{1} \Phi_{17} \Phi_{18}-\frac{160}{27} B r_{1} \beta_{1} \Phi_{13}^{2} \Phi_{17} \Phi_{18} \\
& \Psi_{16}=-\frac{3}{4} B r_{1} \beta_{1} \Phi_{14}^{2} \Phi_{15}^{2}+B r_{1} \beta_{1} \Phi_{12} \Phi_{15}^{3}-\frac{3}{8} B r_{1} \beta_{1} \Phi_{14}^{3} \Phi_{16}+\frac{9}{2} B r_{1} \beta_{1} \Phi_{12} \Phi_{14} \Phi_{15} \Phi_{16}+\frac{9}{2} B r_{1} \beta_{1} \Phi_{13} \Phi_{15}^{2} \Phi_{16}-\frac{27}{16} \\
& \cdot B r_{1} \beta_{1} \Phi_{12}^{2} \Phi_{16}^{2}+\frac{27}{8} B r_{1} \beta_{1} \Phi_{13} \Phi_{14} \Phi_{16}^{2}+\frac{3}{2} B r_{1} \beta_{1} \Phi_{12} \Phi_{14}^{2} \Phi_{17}-3 B r_{1} \beta_{1} \Phi_{12}^{2} \Phi_{15} \Phi_{17}+6 B r_{1} \beta_{1} \Phi_{13} \Phi_{14} \Phi_{15} \Phi_{17} \\
& -9 B r_{1} \beta_{1} \Phi_{12} \Phi_{13} \Phi_{16} \Phi_{17}-\frac{1}{4} B r_{1} \Phi_{17}^{2}-3 B r_{1} \beta_{1} \Phi_{13}^{2} \Phi_{17}^{2}-\frac{15}{8} B r_{1} \beta_{1} \Phi_{12}^{2} \Phi_{14} \Phi_{18}+\frac{15}{8} B r_{1} \beta_{1} \Phi_{13} \Phi_{14}^{2} \Phi_{18}-\frac{15}{2} \\
& \cdot B r_{1} \beta_{1} \Phi_{12} \Phi_{13} \Phi_{15} \Phi_{18}-\frac{15}{32} B r_{1} \Phi_{16} \Phi_{18}-\frac{45}{8} B r_{1} \beta_{1} \Phi_{13}^{2} \Phi_{16} \Phi_{18} \\
& \Psi_{17}=-\frac{16}{49} B r_{1} \beta_{1} \Phi_{14}^{3} \Phi_{15}+\frac{96}{49} B r_{1} \beta_{1} \Phi_{12} \Phi_{14} \Phi_{15}^{2}+\frac{64}{49} B r_{1} \beta_{1} \Phi_{13} \Phi_{15}^{3}+\frac{72}{49} B r_{1} \beta_{1} \Phi_{12} \Phi_{14}^{2} \Phi_{16}-\frac{144}{49} B r_{1} \beta_{1} \Phi_{12}^{2} \Phi_{15} \Phi_{16} \\
& +\frac{288}{49} B r_{1} \beta_{1} \Phi_{13} \Phi_{14} \Phi_{15} \Phi_{16}-\frac{216}{49} B r_{1} \beta_{1} \Phi_{12} \Phi_{13} \Phi_{16}^{2}-\frac{96}{49} B r_{1} \beta_{1} \Phi_{12}^{2} \Phi_{14} \Phi_{17}+\frac{96}{49} B r_{1} \beta_{1} \Phi_{13} \Phi_{14}^{2} \Phi_{17}-\frac{384}{49} \\
& \cdot B r_{1} \beta_{1} \Phi_{12} \Phi_{13} \Phi_{15} \Phi_{17}-\frac{24}{49} B r_{1} \Phi_{16} \Phi_{17}-\frac{288}{49} B r_{1} \beta_{1} \Phi_{13}^{2} \Phi_{16} \Phi_{17}+\frac{40}{49} B r_{1} \beta_{1} \Phi_{12}^{3} \Phi_{18}-\frac{240}{49} B r_{1} \beta_{1} \Phi_{12} \Phi_{13} \Phi_{14} \Phi_{18} \\
& -\frac{20}{49} B r_{1} \Phi_{15} \Phi_{18}-\frac{240}{49} B r_{1} \beta_{1} \Phi_{13}^{2} \Phi_{15} \Phi_{18} \\
& \Psi_{18}=-\frac{1}{18} B r_{1} \beta_{1} \Phi_{14}^{4}+\frac{4}{3} B r_{1} \beta_{1} \Phi_{12} \Phi_{14}^{2} \Phi_{15}-\frac{4}{3} B r_{1} \beta_{1} \Phi_{12}^{2} \Phi_{15}^{2}+\frac{8}{3} B r_{1} \beta_{1} \Phi_{13} \Phi_{14} \Phi_{15}^{2}-2 B r_{1} \beta_{1} \Phi_{12}^{2} \Phi_{14} \Phi_{16} \\
& +2 B r_{1} \beta_{1} \Phi_{13} \Phi_{14}^{2} \Phi_{16}-8 B r_{1} \beta_{1} \Phi_{12} \Phi_{13} \Phi_{15} \Phi_{16}-\frac{1}{4} B r_{1} \Phi_{16}^{2}-3 B r_{1} \beta_{1} \Phi_{13}^{2} \Phi_{16}^{2}+\frac{8}{9} B r_{1} \beta_{1} \Phi_{12}^{3} \Phi_{17}-\frac{16}{3} \\
& \cdot B r_{1} \beta_{1} \Phi_{12} \Phi_{13} \Phi_{14} \Phi_{17}-\frac{4}{9} B r_{1} \Phi_{15} \Phi_{17}-\frac{16}{3} B r_{1} \beta_{1} \Phi_{13}^{2} \Phi_{15} \Phi_{17}+\frac{10}{3} B r_{1} \beta_{1} \Phi_{12}^{2} \Phi_{13} \Phi_{18}-\frac{5}{18} B r_{1} \Phi_{14} \Phi_{18}-\frac{10}{3} \\
& \text { - } B r_{1} \beta_{1} \Phi_{13}^{2} \Phi_{14} \Phi_{18} \\
& \Psi_{19}=\frac{8}{25} B r_{1} \beta_{1} \Phi_{12} \Phi_{14}^{3}-\frac{48}{25} B r_{1} \beta_{1} \Phi_{12}^{2} \Phi_{14} \Phi_{15}+\frac{48}{25} B r_{1} \beta_{1} \Phi_{13} \Phi_{14}^{2} \Phi_{15}-\frac{96}{25} B r_{1} \beta_{1} \Phi_{12} \Phi_{13} \Phi_{15}^{2}+\frac{24}{25} B r_{1} \beta_{1} \Phi_{12}^{3} \Phi_{16} \\
& -\frac{144}{25} B r_{1} \beta_{1} \Phi_{12} \Phi_{13} \Phi_{14} \Phi_{16}-\frac{12}{25} B r_{1} \Phi_{15} \Phi_{16}-\frac{144}{25} B r_{1} \beta_{1} \Phi_{13}^{2} \Phi_{15} \Phi_{16}+\frac{96}{25} B r_{1} \beta_{1} \Phi_{12}^{2} \Phi_{13} \Phi_{17}-\frac{8}{25} B r_{1} \Phi_{14} \Phi_{17}-\frac{96}{25} \\
& \cdot B r_{1} \beta_{1} \Phi_{13}^{2} \Phi_{14} \Phi_{17}+\frac{2}{5} B r_{1} \Phi_{12} \Phi_{18}+\frac{24}{5} B r_{1} \beta_{1} \Phi_{12} \Phi_{13}^{2} \Phi_{18} \\
& \Psi_{20}=-\frac{3}{4} B r_{1} \beta_{1} \Phi_{12}^{2} \Phi_{14}^{2}+\frac{1}{2} B r_{1} \beta_{1} \Phi_{13} \Phi_{14}^{3}+B r_{1} \beta_{1} \Phi_{12}^{3} \Phi_{15}-6 B r_{1} \beta_{1} \Phi_{12} \Phi_{13} \Phi_{14} \Phi_{15}-\frac{1}{4} B r_{1} \Phi_{15}^{2}-3 B r_{1} \beta_{1} \Phi_{13}^{2} \Phi_{15}^{2}+\frac{9}{2} \\
& \cdot B r_{1} \beta_{1} \Phi_{12}^{2} \Phi_{13} \Phi_{16}-\frac{3}{8} B r_{1} \Phi_{14} \Phi_{16}-\frac{9}{2} B r_{1} \beta_{1} \Phi_{13}^{2} \Phi_{14} \Phi_{16}+\frac{1}{2} B r_{1} \Phi_{12} \Phi_{17}+6 B r_{1} \beta_{1} \Phi_{12} \Phi_{13}^{2} \Phi_{17}+\frac{5}{8} B r_{1} \Phi_{13} \Phi_{18}+\frac{5}{2} \\
& \cdot B r_{1} \beta_{1} \Phi_{13}^{3} \Phi_{18} \\
& \Psi_{21}=\frac{8}{9} B r_{1} \beta_{1} \Phi_{12}^{3} \Phi_{14}-\frac{8}{3} B r_{1} \beta_{1} \Phi_{12} \Phi_{13} \Phi_{14}^{2}+\frac{16}{3} B r_{1} \beta_{1} \Phi_{12}^{2} \Phi_{13} \Phi_{15}-\frac{4}{9} B r_{1} \Phi_{14} \Phi_{15}-\frac{16}{3} B r_{1} \beta_{1} \Phi_{13}^{2} \Phi_{14} \Phi_{15}+\frac{2}{3} \\
& \cdot B r_{1} \Phi_{12} \Phi_{16}+8 B r_{1} \beta_{1} \Phi_{12} \Phi_{13}^{2} \Phi_{16}+\frac{8}{9} B r_{1} \Phi_{13} \Phi_{17}+\frac{32}{9} B r_{1} \beta_{1} \Phi_{13}^{3} \Phi_{17} \\
& \Psi_{22}=-\frac{1}{2} B r_{1} \beta_{1} \Phi_{12}^{4}+6 B r_{1} \beta_{1} \Phi_{12}^{2} \Phi_{13} \Phi_{14}-\frac{1}{4} B r_{1} \Phi_{14}^{2}-3 B r_{1} \beta_{1} \Phi_{13}^{2} \Phi_{14}^{2}+B r_{1} \Phi_{12} \Phi_{15}+12 B r_{1} \beta_{1} \Phi_{12} \Phi_{13}^{2} \Phi_{15}+\frac{3}{2} \\
& \cdot B r_{1} \Phi_{13} \Phi_{16}+6 B r_{1} \beta_{1} \Phi_{13}^{3} \Phi_{16}
\end{aligned}
$$




$$
\begin{aligned}
& \Psi_{23}=-8 B r_{1} \beta_{1} \Phi_{12}^{3} \Phi_{13}+2 B r_{1} \Phi_{12} \Phi_{14}+24 B r_{1} \beta_{1} \Phi_{12} \Phi_{13}^{2} \Phi_{14}+4 B r_{1} \Phi_{13} \Phi_{15}+16 B r_{1} \beta_{1} \Phi_{13}^{3} \Phi_{15}, \\
& \Psi_{24}=-2 B r_{1} \Phi_{12} \Phi_{13}-8 B r_{1} \beta_{1} \Phi_{12} \Phi_{13}^{3} \text {, } \\
& \Psi_{25}=-\frac{1}{4} B r_{1} \Phi_{13}^{2}-\frac{1}{2} B r_{1} \beta_{1} \Phi_{13}^{4} \text {, } \\
& \Psi_{26}=-\frac{1}{2} B r_{1} \Phi_{12}^{2}-6 B r_{1} \beta_{1} \Phi_{12}^{2} \Phi_{13}^{2}+B r_{1} \Phi_{13} \Phi_{14}+4 B r_{1} \beta_{1} \Phi_{13}^{3} \Phi_{14} \\
& \Lambda_{1}=-\frac{625}{242} B r_{2} \beta_{2} \Gamma_{18}^{4} \\
& \Lambda_{2}=-\frac{4000}{441} B r_{2} \beta_{2} \Gamma_{17} \Gamma_{18}^{3} \\
& \Lambda_{3}=-12 B r_{2} \beta_{2} \Gamma_{17}^{2} \Gamma_{18}^{2}-\frac{15}{2} B r_{2} \beta_{2} \Gamma_{16} \Gamma_{18}^{3} \text {, } \\
& \Lambda_{4}=-12 B r_{2} \beta_{2} \Gamma_{17}^{2} \Gamma_{18}^{2}-\frac{15}{2} B r_{2} \beta_{2} \Gamma_{16} \Gamma_{18}^{3} \text {, } \\
& \Lambda_{5}=-\frac{2560}{361} B r_{2} \beta_{2} \Gamma_{17}^{3} \Gamma_{18}-\frac{7200}{361} B r_{2} \beta_{2} \Gamma_{16} \Gamma_{17} \Gamma_{18}^{2}-\frac{2000}{361} B r_{2} \beta_{2} \Gamma_{15} \Gamma_{18}^{3}, \\
& \Lambda_{6}=-\frac{128}{81} B r_{2} \beta_{2} \Gamma_{17}^{4}-\frac{160}{9} B r_{2} \beta_{2} \Gamma_{16} \Gamma_{17}^{2} \Gamma_{18}-\frac{25}{3} B r_{2} \beta_{2} \Gamma_{16}^{2} \Gamma_{18}^{2}-\frac{400}{27} B r_{2} \beta_{2} \Gamma_{15} \Gamma_{17} \Gamma_{18}^{2}-\frac{250}{81} B r_{2} \beta_{2} \Gamma_{14} \Gamma_{18}^{3} \text {, } \\
& \Lambda_{7}=-\frac{1536}{289} B r_{2} \beta_{2} \Gamma_{16} \Gamma_{17}^{3}-\frac{4320}{289} B r_{2} \beta_{2} \Gamma_{16}^{2} \Gamma_{17} \Gamma_{18}-\frac{3840}{289} B r_{2} \beta_{2} \Gamma_{15} \Gamma_{17}^{2} \Gamma_{18}-\frac{3600}{289} B r_{2} \beta_{2} \Gamma_{15} \Gamma_{16} \Gamma_{18}^{2}-\frac{2400}{289} B r_{2} \beta_{2} \Gamma_{14} \Gamma_{17} \Gamma_{18}^{2} \\
& +\frac{1000}{289} \mathrm{Br}_{2} \beta_{2} \Gamma_{12} \Gamma_{18}^{3} \\
& \Lambda_{8}=-\frac{27}{4} B r_{2} \beta_{2} \Gamma_{16}^{2} \Gamma_{17}^{2}-4 B r_{2} \beta_{2} \Gamma_{15} \Gamma_{17}^{3}-\frac{135}{32} B r_{2} \beta_{2} \Gamma_{16}^{3} \Gamma_{18}-\frac{45}{2} B r_{2} \beta_{2} \Gamma_{15} \Gamma_{16} \Gamma_{17} \Gamma_{18}-\frac{15}{2} B r_{2} \beta_{2} \Gamma_{14} \Gamma_{17}^{2} \Gamma_{18}-\frac{75}{16} \\
& \cdot B r_{2} \beta_{2} \Gamma_{15}^{2} \Gamma_{18}^{2}-\frac{225}{32} B r_{2} \beta_{2} \Gamma_{14} \Gamma_{16} \Gamma_{18}^{2}+\frac{75}{8} B r_{2} \beta_{2} \Gamma_{12} \Gamma_{17} \Gamma_{18}^{2}+\frac{125}{32} B r_{2} \beta_{2} \Gamma_{13} \Gamma_{18}^{3} \text {, } \\
& \Lambda_{9}=-\frac{96}{25} B r_{2} \beta_{2} \Gamma_{16}^{3} \Gamma_{17}-\frac{256}{25} B r_{2} \beta_{2} \Gamma_{15} \Gamma_{16} \Gamma_{17}^{2}-\frac{512}{225} B r_{2} \beta_{2} \Gamma_{14} \Gamma_{17}^{3}-\frac{48}{5} B r_{2} \beta_{2} \Gamma_{15} \Gamma_{16}^{2} \Gamma_{18}-\frac{128}{15} B r_{2} \beta_{2} \Gamma_{15}^{2} \Gamma_{17} \Gamma_{18}-\frac{64}{5} \\
& \cdot B r_{2} \beta_{2} \Gamma_{14} \Gamma_{16} \Gamma_{17} \Gamma_{18}+\frac{128}{15} B r_{2} \beta_{2} \Gamma_{12} \Gamma_{17}^{2} \Gamma_{18}-\frac{16}{3} B r_{2} \beta_{2} \Gamma_{14} \Gamma_{15} \Gamma_{18}^{2}+8 B r_{2} \beta_{2} \Gamma_{12} \Gamma_{16} \Gamma_{18}^{2}+\frac{32}{3} B r_{2} \beta_{2} \Gamma_{13} \Gamma_{17} \Gamma_{18}^{2} \text {, } \\
& \Lambda_{10}=-\frac{81}{98} B r_{2} \beta_{2} \Gamma_{16}^{4}-\frac{432}{49} B r_{2} \beta_{2} \Gamma_{15} \Gamma_{16}^{2} \Gamma_{17}-\frac{192}{49} B r_{2} \beta_{2} \Gamma_{15}^{2} \Gamma_{17}^{2}-\frac{288}{49} B r_{2} \beta_{2} \Gamma_{14} \Gamma_{16} \Gamma_{17}^{2}+\frac{128}{49} B r_{2} \beta_{2} \Gamma_{12} \Gamma_{17}^{3}-\frac{360}{49} \\
& \cdot B r_{2} \beta_{2} \Gamma_{15}^{2} \Gamma_{16} \Gamma_{18}-\frac{270}{49} B r_{2} \beta_{2} \Gamma_{14} \Gamma_{16}^{2} \Gamma_{18}-\frac{480}{49} B r_{2} \beta_{2} \Gamma_{14} \Gamma_{15} \Gamma_{17} \Gamma_{18}+\frac{720}{49} B r_{2} \beta_{2} \Gamma_{12} \Gamma_{16} \Gamma_{17} \Gamma_{18}+\frac{480}{49} B r_{2} \beta_{2} \Gamma_{13} \Gamma_{17}^{2} \Gamma_{18}-\frac{75}{49} \\
& \cdot B r_{2} \beta_{2} \Gamma_{14}^{2} \Gamma_{18}^{2}+\frac{300}{49} B r_{2} \beta_{2} \Gamma_{12} \Gamma_{15} \Gamma_{18}^{2}+\frac{450}{49} B r_{2} \beta_{2} \Gamma_{13} \Gamma_{16} \Gamma_{18}^{2} \\
& \Lambda_{11}=-\frac{432}{169} B r_{2} \beta_{2} \Gamma_{15} \Gamma_{16}^{3}-\frac{1152}{169} B r_{2} \beta_{2} \Gamma_{15}^{2} \Gamma_{16} \Gamma_{17}-\frac{864}{169} B r_{2} \beta_{2} \Gamma_{14} \Gamma_{16}^{2} \Gamma_{17}-\frac{768}{169} B r_{2} \beta_{2} \Gamma_{14} \Gamma_{15} \Gamma_{17}^{2}+\frac{1152}{169} B r_{2} \beta_{2} \Gamma_{12} \Gamma_{16} \Gamma_{17}^{2} \\
& +\frac{512}{169} B r_{2} \beta_{2} \Gamma_{13} \Gamma_{17}^{3}-\frac{320}{169} B r_{2} \beta_{2} \Gamma_{15}^{3} \Gamma_{18}-\frac{1440}{169} B r_{2} \beta_{2} \Gamma_{14} \Gamma_{15} \Gamma_{16} \Gamma_{18}+\frac{1080}{169} B r_{2} \beta_{2} \Gamma_{12} \Gamma_{16}^{2} \Gamma_{18}-\frac{480}{169} B r_{2} \beta_{2} \Gamma_{14}^{2} \Gamma_{17} \Gamma_{18} \\
& +\frac{1920}{169} B r_{2} \beta_{2} \Gamma_{12} \Gamma_{15} \Gamma_{17} \Gamma_{18}+\frac{2880}{169} B r_{2} \beta_{2} \Gamma_{13} \Gamma_{16} \Gamma_{17} \Gamma_{18}+\frac{600}{169} B r_{2} \beta_{2} \Gamma_{12} \Gamma_{14} \Gamma_{18}^{2}+\frac{1200}{169} B r_{2} \beta_{2} \Gamma_{13} \Gamma_{15} \Gamma_{18}^{2} \text {, } \\
& \Lambda_{12}=-3 B r_{2} \beta_{2} \Gamma_{15}^{2} \Gamma_{16}^{2}-\frac{3}{2} B r_{2} \beta_{2} \Gamma_{14} \Gamma_{16}^{3}-\frac{16}{9} B r_{2} \beta_{2} \Gamma_{15}^{3} \Gamma_{17}-8 B r_{2} \beta_{2} \Gamma_{14} \Gamma_{15} \Gamma_{16} \Gamma_{17}+6 B r_{2} \beta_{2} \Gamma_{12} \Gamma_{16}^{2} \Gamma_{17}-\frac{4}{3} B r_{2} \beta_{2} \Gamma_{14}^{2} \Gamma_{17}^{2}+\frac{16}{3} \\
& \cdot B r_{2} \beta_{2} \Gamma_{12} \Gamma_{15} \Gamma_{17}^{2}+8 B r_{2} \beta_{2} \Gamma_{13} \Gamma_{16} \Gamma_{17}^{2}-\frac{10}{3} B r_{2} \beta_{2} \Gamma_{14} \Gamma_{15}^{2} \Gamma_{18}-\frac{5}{2} B r_{2} \beta_{2} \Gamma_{14}^{2} \Gamma_{16} \Gamma_{18}+10 B r_{2} \beta_{2} \Gamma_{12} \Gamma_{15} \Gamma_{16} \Gamma_{18}+\frac{15}{2}
\end{aligned}
$$


$\cdot B r_{2} \beta_{2} \Gamma_{13} \Gamma_{16}^{2} \Gamma_{18}+\frac{20}{3} B r_{2} \beta_{2} \Gamma_{12} \Gamma_{14} \Gamma_{17} \Gamma_{18}+\frac{40}{3} B r_{2} \beta_{2} \Gamma_{13} \Gamma_{15} \Gamma_{17} \Gamma_{18}-\frac{25}{12} B r_{2} \beta_{2} \Gamma_{12}^{2} \Gamma_{18}^{2}+\frac{25}{6} B r_{2} \beta_{2} \Gamma_{13} \Gamma_{14} \Gamma_{18}^{2}$, $\Lambda_{13}=-\frac{192}{121} B r_{2} \beta_{2} \Gamma_{15}^{3} \Gamma_{16}-\frac{432}{121} B r_{2} \beta_{2} \Gamma_{14} \Gamma_{15} \Gamma_{16}^{2}+\frac{216}{121} B r_{2} \beta_{2} \Gamma_{12} \Gamma_{16}^{3}-\frac{384}{121} B r_{2} \beta_{2} \Gamma_{14} \Gamma_{15}^{2} \Gamma_{17}-\frac{288}{121} B r_{2} \beta_{2} \Gamma_{14}^{2} \Gamma_{16} \Gamma_{17}+\frac{1152}{121}$ $\cdot B r_{2} \beta_{2} \Gamma_{12} \Gamma_{15} \Gamma_{16} \Gamma_{17}+\frac{864}{121} B r_{2} \beta_{2} \Gamma_{13} \Gamma_{16}^{2} \Gamma_{17}+\frac{384}{121} B r_{2} \beta_{2} \Gamma_{12} \Gamma_{14} \Gamma_{17}^{2}+\frac{768}{121} B r_{2} \beta_{2} \Gamma_{13} \Gamma_{15} \Gamma_{17}^{2}-\frac{240}{121} B r_{2} \beta_{2} \Gamma_{14}^{2} \Gamma_{15} \Gamma_{18}+\frac{480}{121}$ $\cdot B r_{2} \beta_{2} \Gamma_{12} \Gamma_{15}^{2} \Gamma_{18}+\frac{720}{121} B r_{2} \beta_{2} \Gamma_{12} \Gamma_{14} \Gamma_{16} \Gamma_{18}+\frac{1440}{121} B r_{2} \beta_{2} \Gamma_{13} \Gamma_{15} \Gamma_{16} \Gamma_{18}-\frac{480}{121} B r_{2} \beta_{2} \Gamma_{12}^{2} \Gamma_{17} \Gamma_{18}+\frac{960}{121} B r_{2} \beta_{2} \Gamma_{13} \Gamma_{14} \Gamma_{17} \Gamma_{18}$ $-\frac{600}{121} B r_{2} \beta_{2} \Gamma_{12} \Gamma_{13} \Gamma_{18}^{2}$

$\Lambda_{14}=-\frac{8}{25} B r_{2} \beta_{2} \Gamma_{15}^{4}-\frac{72}{25} B r_{2} \beta_{2} \Gamma_{14} \Gamma_{15}^{2} \Gamma_{16}-\frac{27}{25} B r_{2} \beta_{2} \Gamma_{14}^{2} \Gamma_{16}^{2}+\frac{108}{25} B r_{2} \beta_{2} \Gamma_{12} \Gamma_{15} \Gamma_{16}^{2}+\frac{54}{25} B r_{2} \beta_{2} \Gamma_{13} \Gamma_{16}^{3}-\frac{48}{25}$

$\cdot B r_{2} \beta_{2} \Gamma_{14}^{2} \Gamma_{15} \Gamma_{17}+\frac{96}{25} B r_{2} \beta_{2} \Gamma_{12} \Gamma_{15}^{2} \Gamma_{17}+\frac{144}{25} B r_{2} \beta_{2} \Gamma_{12} \Gamma_{14} \Gamma_{16} \Gamma_{17}+\frac{288}{25} B r_{2} \beta_{2} \Gamma_{13} \Gamma_{15} \Gamma_{16} \Gamma_{17}-\frac{48}{25} B r_{2} \beta_{2} \Gamma_{12}^{2} \Gamma_{17}^{2}+\frac{96}{25}$

$\cdot B r_{2} \beta_{2} \Gamma_{13} \Gamma_{14} \Gamma_{17}^{2}-\frac{2}{5} B r_{2} \beta_{2} \Gamma_{14}^{3} \Gamma_{18}+\frac{24}{5} B r_{2} \beta_{2} \Gamma_{12} \Gamma_{14} \Gamma_{15} \Gamma_{18}+\frac{24}{5} B r_{2} \beta_{2} \Gamma_{13} \Gamma_{15}^{2} \Gamma_{18}-\frac{18}{5} B r_{2} \beta_{2} \Gamma_{12}^{2} \Gamma_{16} \Gamma_{18}+\frac{36}{5}$

$\cdot B r_{2} \beta_{2} \Gamma_{13} \Gamma_{14} \Gamma_{16} \Gamma_{18}-\frac{48}{5} B r_{2} \beta_{2} \Gamma_{12} \Gamma_{13} \Gamma_{17} \Gamma_{18}-\frac{1}{4} B r_{2} \Gamma_{18}^{2}-3 B r_{2} \beta_{2} \Gamma_{13}^{2} \Gamma_{18}^{2}$, $\Lambda_{15}=-\frac{64}{81} B r_{2} \beta_{2} \Gamma_{14} \Gamma_{15}^{3}-\frac{16}{9} B r_{2} \beta_{2} \Gamma_{14}^{2} \Gamma_{15} \Gamma_{16}+\frac{32}{9} B r_{2} \beta_{2} \Gamma_{12} \Gamma_{15}^{2} \Gamma_{16}+\frac{8}{3} B r_{2} \beta_{2} \Gamma_{12} \Gamma_{14} \Gamma_{16}^{2}+\frac{16}{3} B r_{2} \beta_{2} \Gamma_{13} \Gamma_{15} \Gamma_{16}^{2}-\frac{32}{81}$ $\cdot B r_{2} \beta_{2} \Gamma_{14}^{3} \Gamma_{17}+\frac{128}{27} B r_{2} \beta_{2} \Gamma_{12} \Gamma_{14} \Gamma_{15} \Gamma_{17}+\frac{128}{27} B r_{2} \beta_{2} \Gamma_{13} \Gamma_{15}^{2} \Gamma_{17}-\frac{32}{9} B r_{2} \beta_{2} \Gamma_{12}^{2} \Gamma_{16} \Gamma_{17}+\frac{64}{9} B r_{2} \beta_{2} \Gamma_{13} \Gamma_{14} \Gamma_{16} \Gamma_{17}-\frac{128}{27}$ $\cdot B r_{2} \beta_{2} \Gamma_{12} \Gamma_{13} \Gamma_{17}^{2}+\frac{40}{27} B r_{2} \beta_{2} \Gamma_{12} \Gamma_{14}^{2} \Gamma_{18}-\frac{80}{27} B r_{2} \beta_{2} \Gamma_{12}^{2} \Gamma_{15} \Gamma_{18}+\frac{160}{27} B r_{2} \beta_{2} \Gamma_{13} \Gamma_{14} \Gamma_{15} \Gamma_{18}-\frac{80}{9} B r_{2} \beta_{2} \Gamma_{12} \Gamma_{13} \Gamma_{16} \Gamma_{18}-\frac{40}{81}$ $\cdot B r_{2} \Gamma_{17} \Gamma_{18}-\frac{160}{27} B r_{2} \beta_{2} \Gamma_{13}^{2} \Gamma_{17} \Gamma_{18}$

$\Lambda_{16}=-\frac{3}{4} B r_{2} \beta_{2} \Gamma_{14}^{2} \Gamma_{15}^{2}+B r_{2} \beta_{2} \Gamma_{12} \Gamma_{15}^{3}-\frac{3}{8} B r_{2} \beta_{2} \Gamma_{14}^{3} \Gamma_{16}+\frac{9}{2} B r_{2} \beta_{2} \Gamma_{12} \Gamma_{14} \Gamma_{15} \Gamma_{16}+\frac{9}{2} B r_{2} \beta_{2} \Gamma_{13} \Gamma_{15}^{2} \Gamma_{16}-\frac{27}{16} B r_{2} \beta_{2} \Gamma_{12}^{2} \Gamma_{16}^{2}+\frac{27}{8}$

$\cdot B r_{2} \beta_{2} \Gamma_{13} \Gamma_{14} \Gamma_{16}^{2}+\frac{3}{2} B r_{2} \beta_{2} \Gamma_{12} \Gamma_{14}^{2} \Gamma_{17}-3 B r_{2} \beta_{2} \Gamma_{12}^{2} \Gamma_{15} \Gamma_{17}+6 B r_{2} \beta_{2} \Gamma_{13} \Gamma_{14} \Gamma_{15} \Gamma_{17}-9 B r_{2} \beta_{2} \Gamma_{12} \Gamma_{13} \Gamma_{16} \Gamma_{17}-\frac{1}{4} B r_{2} \Gamma_{17}^{2}$

$-3 B r_{2} \beta_{2} \Gamma_{13}^{2} \Gamma_{17}^{2}-\frac{15}{8} B r_{2} \beta_{2} \Gamma_{12}^{2} \Gamma_{14} \Gamma_{18}+\frac{15}{8} B r_{2} \beta_{2} \Gamma_{13} \Gamma_{14}^{2} \Gamma_{18}-\frac{15}{2} B r_{2} \beta_{2} \Gamma_{12} \Gamma_{13} \Gamma_{15} \Gamma_{18}-\frac{15}{32} B r_{2} \Gamma_{16} \Gamma_{18}-\frac{45}{8}$

$\cdot B r_{2} \beta_{2} \Gamma_{13}^{2} \Gamma_{16} \Gamma_{18}$

$\Lambda_{17}=-\frac{16}{49} B r_{2} \beta_{2} \Gamma_{14}^{3} \Gamma_{15}+\frac{96}{49} B r_{2} \beta_{2} \Gamma_{12} \Gamma_{14} \Gamma_{15}^{2}+\frac{64}{49} B r_{2} \beta_{2} \Gamma_{13} \Gamma_{15}^{3}+\frac{72}{49} B r_{2} \beta_{2} \Gamma_{12} \Gamma_{14}^{2} \Gamma_{16}-\frac{144}{49} B r_{2} \beta_{2} \Gamma_{12}^{2} \Gamma_{15} \Gamma_{16}+\frac{288}{49}$

- $B r_{2} \beta_{2} \Gamma_{13} \Gamma_{14} \Gamma_{15} \Gamma_{16}-\frac{216}{49} B r_{2} \beta_{2} \Gamma_{12} \Gamma_{13} \Gamma_{16}^{2}-\frac{96}{49} B r_{2} \beta_{2} \Gamma_{12}^{2} \Gamma_{14} \Gamma_{17}+\frac{96}{49} B r_{2} \beta_{2} \Gamma_{13} \Gamma_{14}^{2} \Gamma_{17}-\frac{384}{49} B r_{2} \beta_{2} \Gamma_{12} \Gamma_{13} \Gamma_{15} \Gamma_{17}-\frac{24}{49}$

$\cdot B r_{2} \Gamma_{16} \Gamma_{17}-\frac{288}{49} B r_{2} \beta_{2} \Gamma_{13}^{2} \Gamma_{16} \Gamma_{17}+\frac{40}{49} B r_{2} \beta_{2} \Gamma_{12}^{3} \Gamma_{18}-\frac{240}{49} B r_{2} \beta_{2} \Gamma_{12} \Gamma_{13} \Gamma_{14} \Gamma_{18}-\frac{20}{49} B r_{2} \Gamma_{15} \Gamma_{18}-\frac{240}{49} B r_{2} \beta_{2} \Gamma_{13}^{2} \Gamma_{15} \Gamma_{18}$, $\Lambda_{18}=-\frac{1}{18} B r_{2} \beta_{2} \Gamma_{14}^{4}+\frac{4}{3} B r_{2} \beta_{2} \Gamma_{12} \Gamma_{14}^{2} \Gamma_{15}-\frac{4}{3} B r_{2} \beta_{2} \Gamma_{12}^{2} \Gamma_{15}^{2}+\frac{8}{3} B r_{2} \beta_{2} \Gamma_{13} \Gamma_{14} \Gamma_{15}^{2}-2 B r_{2} \beta_{2} \Gamma_{12}^{2} \Gamma_{14} \Gamma_{16}+2 B r_{2} \beta_{2} \Gamma_{13} \Gamma_{14}^{2} \Gamma_{16}$ $-8 B r_{2} \beta_{2} \Gamma_{12} \Gamma_{13} \Gamma_{15} \Gamma_{16}-\frac{1}{4} B r_{2} \Gamma_{16}^{2}-3 B r_{2} \beta_{2} \Gamma_{13}^{2} \Gamma_{16}^{2}+\frac{8}{9} B r_{2} \beta_{2} \Gamma_{12}^{3} \Gamma_{17}-\frac{16}{3} B r_{2} \beta_{2} \Gamma_{12} \Gamma_{13} \Gamma_{14} \Gamma_{17}-\frac{4}{9} B r_{2} \Gamma_{15} \Gamma_{17}-\frac{16}{3}$ $\cdot B r_{2} \beta_{2} \Gamma_{13}^{2} \Gamma_{15} \Gamma_{17}+\frac{10}{3} B r_{2} \beta_{2} \Gamma_{12}^{2} \Gamma_{13} \Gamma_{18}-\frac{5}{18} B r_{2} \Gamma_{14} \Gamma_{18}-\frac{10}{3} B r_{2} \beta_{2} \Gamma_{13}^{2} \Gamma_{14} \Gamma_{18}$ $\Lambda_{19}=\frac{8}{25} B r_{2} \beta_{2} \Gamma_{12} \Gamma_{14}^{3}-\frac{48}{25} B r_{2} \beta_{2} \Gamma_{12}^{2} \Gamma_{14} \Gamma_{15}+\frac{48}{25} B r_{2} \beta_{2} \Gamma_{13} \Gamma_{14}^{2} \Gamma_{15}-\frac{96}{25} B r_{2} \beta_{2} \Gamma_{12} \Gamma_{13} \Gamma_{15}^{2}+\frac{24}{25} B r_{2} \beta_{2} \Gamma_{12}^{3} \Gamma_{16}-\frac{144}{25}$ 


$$
\begin{aligned}
& \text { - } B r_{2} \beta_{2} \Gamma_{12} \Gamma_{13} \Gamma_{14} \Gamma_{16}-\frac{12}{25} B r_{2} \Gamma_{15} \Gamma_{16}-\frac{144}{25} B r_{2} \beta_{2} \Gamma_{13}^{2} \Gamma_{15} \Gamma_{16}+\frac{96}{25} B r_{2} \beta_{2} \Gamma_{12}^{2} \Gamma_{13} \Gamma_{17}-\frac{8}{25} B r_{2} \Gamma_{14} \Gamma_{17}-\frac{96}{25} B r_{2} \beta_{2} \Gamma_{13}^{2} \Gamma_{14} \Gamma_{17} \\
& +\frac{2}{5} B r_{2} \Gamma_{12} \Gamma_{18}+\frac{24}{5} B r_{2} \beta_{2} \Gamma_{12} \Gamma_{13}^{2} \Gamma_{18} \\
& \Lambda_{20}=-\frac{3}{4} B r_{2} \beta_{2} \Gamma_{12}^{2} \Gamma_{14}^{2}+\frac{1}{2} B r_{2} \beta_{2} \Gamma_{13} \Gamma_{14}^{3}+B r_{2} \beta_{2} \Gamma_{12}^{3} \Gamma_{15}-6 B r_{2} \beta_{2} \Gamma_{12} \Gamma_{13} \Gamma_{14} \Gamma_{15}-\frac{1}{4} B r_{2} \Gamma_{15}^{2}-3 B r_{2} \beta_{2} \Gamma_{13}^{2} \Gamma_{15}^{2}+\frac{9}{2} \\
& \cdot B r_{2} \beta_{2} \Gamma_{12}^{2} \Gamma_{13} \Gamma_{16}-\frac{3}{8} B r_{2} \Gamma_{14} \Gamma_{16}-\frac{9}{2} B r_{2} \beta_{2} \Gamma_{13}^{2} \Gamma_{14} \Gamma_{16}+\frac{1}{2} B r_{2} \Gamma_{12} \Gamma_{17}+6 B r_{2} \beta_{2} \Gamma_{12} \Gamma_{13}^{2} \Gamma_{17}+\frac{5}{8} B r_{2} \Gamma_{13} \Gamma_{18}+\frac{5}{2} B r_{2} \beta_{2} \Gamma_{13}^{3} \Gamma_{18} \text {, } \\
& \Lambda_{21}=\frac{8}{9} B r_{2} \beta_{2} \Gamma_{12}^{3} \Gamma_{14}-\frac{8}{3} B r_{2} \beta_{2} \Gamma_{12} \Gamma_{13} \Gamma_{14}^{2}+\frac{16}{3} B r_{2} \beta_{2} \Gamma_{12}^{2} \Gamma_{13} \Gamma_{15}-\frac{4}{9} B r_{2} \Gamma_{14} \Gamma_{15}-\frac{16}{3} B r_{2} \beta_{2} \Gamma_{13}^{2} \Gamma_{14} \Gamma_{15}+\frac{2}{3} B r_{2} \Gamma_{12} \Gamma_{16} \\
& +8 B r_{2} \beta_{2} \Gamma_{12} \Gamma_{13}^{2} \Gamma_{16}+\frac{8}{9} B r_{2} \Gamma_{13} \Gamma_{17}+\frac{32}{9} B r_{2} \beta_{2} \Gamma_{13}^{3} \Gamma_{17} \\
& \Lambda_{22}=-\frac{1}{2} B r_{2} \beta_{2} \Gamma_{12}^{4}+6 B r_{2} \beta_{2} \Gamma_{12}^{2} \Gamma_{13} \Gamma_{14}-\frac{1}{4} B r_{2} \Gamma_{14}^{2}-3 B r_{2} \beta_{2} \Gamma_{13}^{2} \Gamma_{14}^{2}+B r_{2} \Gamma_{12} \Gamma_{15}+12 B r_{2} \beta_{2} \Gamma_{12} \Gamma_{13}^{2} \Gamma_{15}+\frac{3}{2} B r_{2} \Gamma_{13} \Gamma_{16} \\
& +6 \mathrm{Br}_{2} \beta_{2} \Gamma_{13}^{3} \Gamma_{16} \\
& \Lambda_{23}=-8 B r_{2} \beta_{2} \Gamma_{12}^{3} \Gamma_{13}+2 B r_{2} \Gamma_{12} \Gamma_{14}+24 B r_{2} \beta_{2} \Gamma_{12} \Gamma_{13}^{2} \Gamma_{14}+4 B r_{2} \Gamma_{13} \Gamma_{15}+16 B r_{2} \beta_{2} \Gamma_{13}^{3} \Gamma_{15} \text {, } \\
& \Lambda_{24}=-2 B r_{2} \Gamma_{12} \Gamma_{13}-8 B r_{2} \beta_{2} \Gamma_{12} \Gamma_{13}^{3} \text {, } \\
& \Lambda_{25}=-\frac{1}{4} B r_{2} \Gamma_{13}^{2}-\frac{1}{2} B r_{2} \beta_{2} \Gamma_{13}^{4} \text {, } \\
& \Lambda_{26}=-\frac{1}{2} B r_{2} \Gamma_{12}^{2}-6 B r_{2} \beta_{2} \Gamma_{12}^{2} \Gamma_{13}^{2}+B r_{2} \Gamma_{13} \Gamma_{14}+4 B r_{2} \beta_{2} \Gamma_{13}^{3} \Gamma_{14} \text {, } \\
& k_{1}=\frac{U-1}{\ln \Omega}-\left(\frac{p_{2}}{3 p_{3}}-\frac{2^{1 / 3}\left(-p_{2}^{2}+3 p_{1} p_{3}\right)}{3 p_{3}\left(-2 p_{2}^{3}+9 p_{1} p_{2} p_{3}-27 p_{3}^{2} p_{4}+\sqrt{4\left(-p_{2}^{2}+3 p_{1} p_{3}\right)^{3}+\left(-2 p_{2}^{3}+9 p_{1} p_{2} p_{3}-27 p_{3}^{2} p_{4}\right)^{2}}\right)^{1 / 3}}\right. \\
& \left.+\frac{\left(-2 p_{2}^{3}+9 p_{1} p_{2} p_{3}-27 p_{3}^{2} p_{4}+\sqrt{4\left(-p_{2}^{2}+3 p_{1} p_{3}\right)^{3}+\left(-2 p_{2}^{3}+9 p_{1} p_{2} p_{3}-27 p_{3}^{2} p_{4}\right)^{2}}\right)^{1 / 3}}{32^{1 / 3} p_{3}}\right)\left(\frac{\ln \delta}{\ln \Omega}-1\right), \\
& k_{2}=-\frac{p_{2}}{3 p_{3}}-\frac{2^{1 / 3}\left(-p_{2}^{2}+3 p_{1} p_{3}\right)}{3 p_{3}\left(-2 p_{2}^{3}+9 p_{1} p_{2} p_{3}-27 p_{3}^{2} p_{4}+\sqrt{4\left(-p_{2}^{2}+3 p_{1} p_{3}\right)^{3}+\left(-2 p_{2}^{3}+9 p_{1} p_{2} p_{3}-27 p_{3}^{2} p_{4}\right)^{2}}\right)^{1 / 3}} \\
& +\frac{\left(-2 p_{2}^{3}+9 p_{1} p_{2} p_{3}-27 p_{3}^{2} p_{4}+\sqrt{4\left(-p_{2}^{2}+3 p_{1} p_{3}\right)^{3}+\left(-2 p_{2}^{3}+9 p_{1} p_{2} p_{3}-27 p_{3}^{2} p_{4}\right)^{2}}\right)^{1 / 3}}{32^{1 / 3} p_{3}} .
\end{aligned}
$$

\section{Competing Interests}

The authors declare that they have no competing interests.

\section{References}

[1] A. M. Siddiqui, T. Haroon, and S. Irum, "Torsional flow of third grade fluid using modified homotopy perturbation method,"
Computers \& Mathematics with Applications, vol. 58, no. 11-12, pp. 2274-2285, 2009.

[2] A. M. Siddiqui, A. Zeb, Q. K. Ghori, and A. Benharbit, "Homotopy perturbation method for heat transfer flow of a third grade fluid between parallel plates," Chaos, Solitons \& Fractals, vol. 36, no. 1, pp. 182-192, 2008.

[3] S. Islam, R. A. Shah, and I. Ali, "Optimal homotopy asymptotic solutions of couette and poiseuille flows of a third grade fluid 
with heat transfer analysis," Internatioal Journal of Non-Linear Sciences and Numerical Simulation, vol. 11, pp. 389-400, 2010.

[4] Y. Aksoy and M. Pakdemirli, "Approximate analytical solutions for flow of a third-grade fluid through a parallel-plate channel filled with a porous medium," Transport in Porous Media, vol. 83, no. 2, pp. 375-395, 2010.

[5] A. M. Siddiqui, R. Mahmood, and Q. K. Ghori, "Thin film flow of a third grade fluid on a moving belt by He's homotopy perturbation method," International Journal of Nonlinear Sciences and Numerical Simulation, vol. 7, no. 1, pp. 7-14, 2006.

[6] A. M. Siddiqui, R. Mahmood, and Q. K. Ghori, "Homotopy perturbation method for thin film flow of a third grade fluid down an inclined plane," Chaos, Solitons \& Fractals, vol. 35, no. 1, pp. 140-147, 2008.

[7] G. K. Batchelor, An Introduction to Fluid Dynamics, Cambridge University Press, Cambridge, UK, 1999.

[8] M. Ishii, Thermo-Fluid Dynamic Theory of Two-Phase Flow, Eyrolles, Paris, France, 1975.

[9] M. Akai, A. Inoue, and S. Aoki, "The prediction of stratified two-phase flow with a two-equation model of turbulence," International Journal of Multiphase Flow, vol. 7, no. 1, pp. 21-39, 1981.

[10] R. A. Shah, S. Islam, A. M. Siddiqui, and T. Haroon, "Heat transfer by laminar flow of an elastico-viscous fluid in posttreatment analysis of wire coating with linearly varying temperature along the coated wire," Heat and Mass Transfer, vol. 48, no. 6, pp. 903914, 2012.

[11] R. A. Shah, S. Islam, A. M. Siddiqui, and T. Haroon, "Optimal homotopy asymptotic method solution of unsteady second grade fluid in wire coating analysis," Journal of the Korean Society for Industrial and Applied Mathematics, vol. 15, no. 3, pp. 201-222, 2011.

[12] R. A. Shah, S. Islam, and A. M. Siddiqui, "Exact solution of a differential equation arising in the wire coating analysis of an unsteady second grade fluid," Mathematical and Computer Modelling, vol. 57, no. 5-6, pp. 1284-1288, 2013.

[13] R. A. Shah, S. Islam, M. Ellahi, T. Haroon, and A. M. Siddiqui, "Analytical solutions for heat transfer flows of a third Grade fluid in case of post-treatment of wire coating," International Journal of the Physical Sciences, vol. 6, pp. 4213-4223, 2011.

[14] K. Kim, H. S. Kwak, S. H. Park, and Y. S. Lee, "Theoretical prediction on double-layer coating in wet-on-wet optical fiber coating process," Journal of Coatings Technology Research, vol. 8, no. 1, pp. 35-44, 2011.

[15] K. J. Kim and H. S. Kwak, "Analytic study of non-Newtonian double layer coating liquid flows in optical fiber manufacturing," Applied Mechanics and Materials, vol. 224, pp. 260-263, 2012.

[16] Zeeshan, R. A. Shah, S. Islam, and A. M. Siddique, "Doublelayer optical fiber coating using viscoelastic phan thien tanner fluid," New York Science Journal, vol. 6, no. 10, pp. 66-73, 2013.

[17] Zeeshan, S. Islam, R. A. Shah, I. Khan, and T. Gul, "Exact solution of PTT fluid in optical fiber coating analysis using two-layer coating flow," Journal of Applied Environmental and Biological Sciences, vol. 5, pp. 96-105, 2015.

[18] Zeeshan, R. A. Shah, I. Khan, T. Gul, and P. Gaskel, "Doublelayer optical fiber coating analysis by withdrawal from a bath of oldroyd 8-constant fluid," Journal of Applied Environmental and Biological Sciences, vol. 5, pp. 36-51, 2015.

[19] Z. Khan, S. Islam, R. A. Shah, and I. Khan, "Flow and heat transfer of two immiscible fluids in double layer optical fiber coating," Journal of Coatings Technology and Research, 2016.
[20] T. Gul, R. A. Shah, S. Islam, and M. Arif, "MHD thin film flows of a third grade fluid on a vertical belt with slip boundary conditions," Journal of Applied Mathematics, vol. 2013, Article ID 707286, 14 pages, 2013.

[21] V. Marinca, N. Herişanu, and I. Nemeş, "Optimal homotopy asymptotic method with application to thin film flow," Central European Journal of Physics, vol. 6, no. 3, pp. 648-653, 2008.

[22] V. Marinca and N. Herişanu, "Application of Optimal Homotopy Asymptotic Method for solving nonlinear equations arising in heat transfer," International Communications in Heat and Mass Transfer, vol. 35, no. 6, pp. 710-715, 2008.

[23] V. Marinca, N. Herisanu, C. Bota, and B. Marinca, "An optimal homotopy asymptotic method applied to the steady flow of a fourth-grade fluid past a porous plate," Applied Mathematics Letters, vol. 22, no. 2, pp. 245-251, 2009.

[24] N. Herisanu, V. Marinca, and G. Madescu, "An analytical approach to non-linear dynamical model of a permanent magnet synchronous generator," Wind Energy, vol. 18, no. 9, pp. 1657-1670, 2015.

[25] N. Herişanu and V. Marinca, "Optimal homotopy perturbation method for a non-conservative dynamical system of a rotating electrical machine," Zeitschrift fur Naturforschung A, vol. 67, no. 8-9, pp. 509-516, 2012.

[26] G. Adomian, "A review of the decomposition method and some recent results for nonlinear equations," Mathematical and Computer Modelling, vol. 13, no. 7, pp. 17-43, 1990.

[27] A.-M. Wazwaz, "Adomian decomposition method for a reliable treatment of the Bratu-type equations," Applied Mathematics and Computation, vol. 166, no. 3, pp. 652-663, 2005.

[28] A.-M. Wazwaz, "Adomian decomposition method for a reliable treatment of the Emden-Fowler equation," Applied Mathematics and Computation, vol. 161, no. 2, pp. 543-560, 2005. 


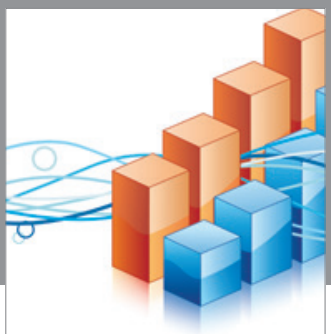

Advances in

Operations Research

vatem alat4

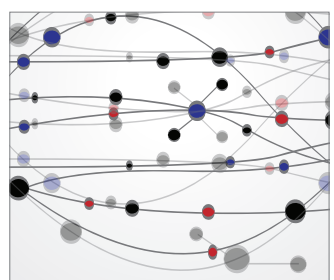

\section{The Scientific} World Journal
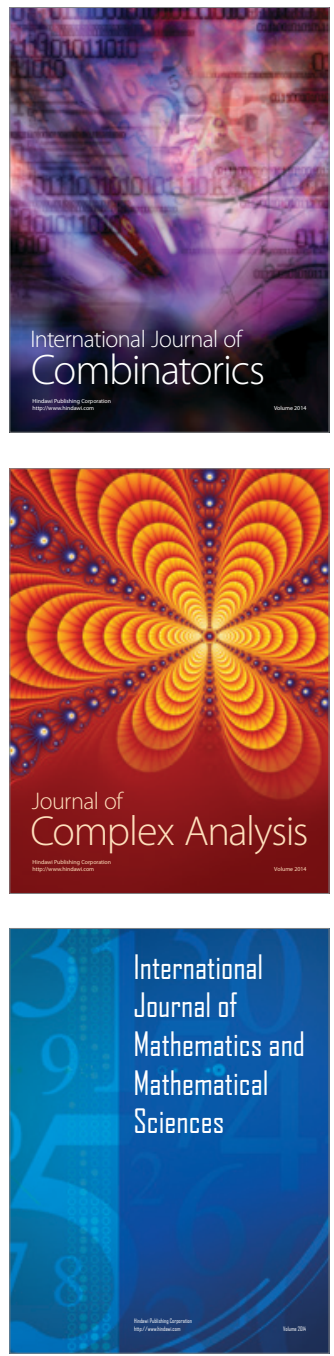
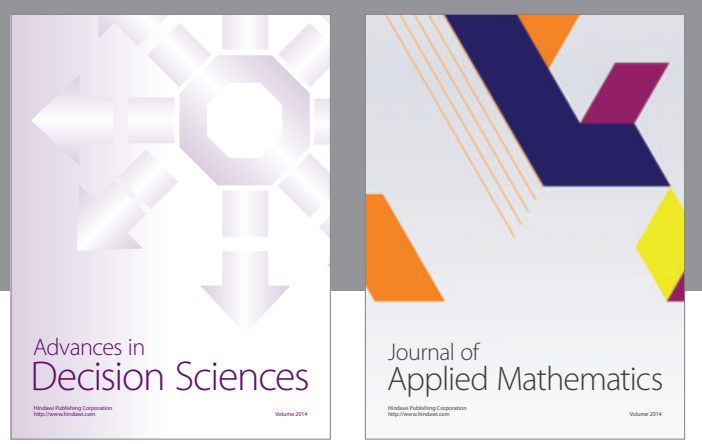

Algebra

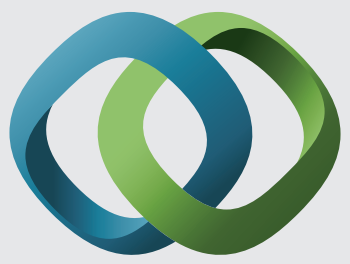

\section{Hindawi}

Submit your manuscripts at

http://www.hindawi.com
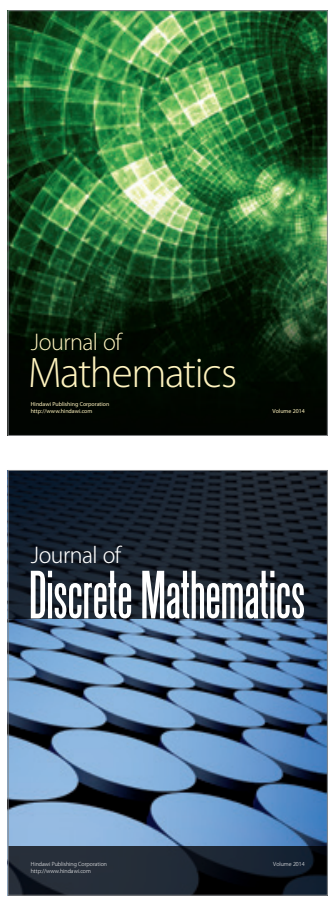

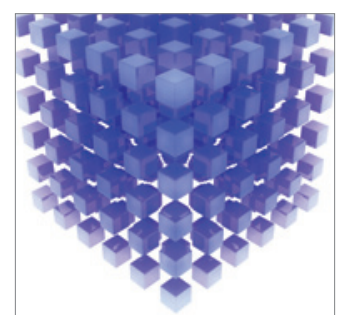

Mathematical Problems in Engineering
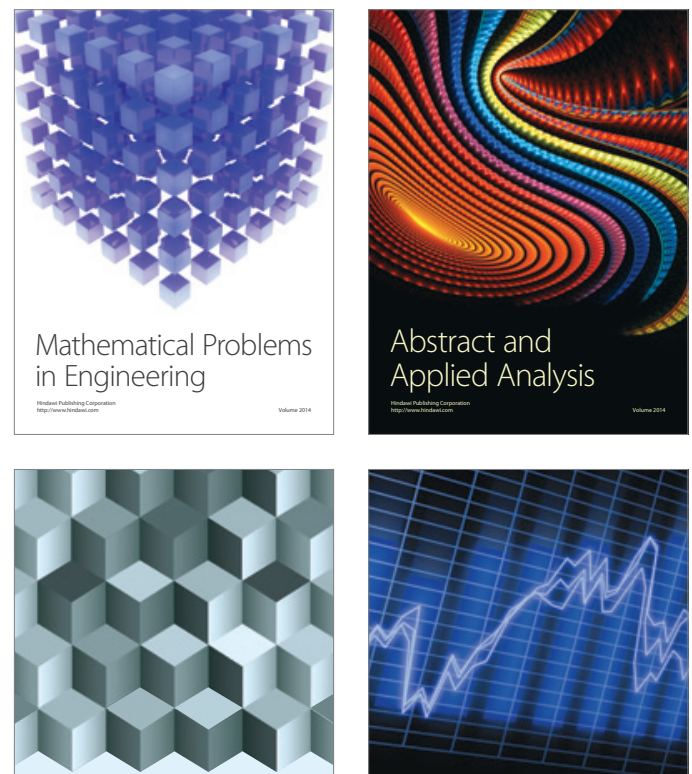

Journal of

Function Spaces

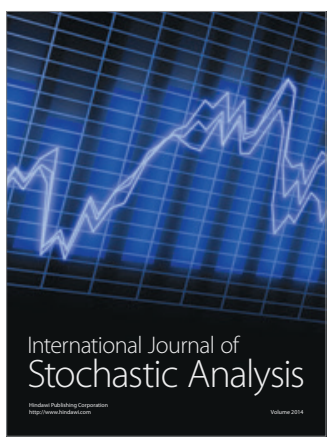

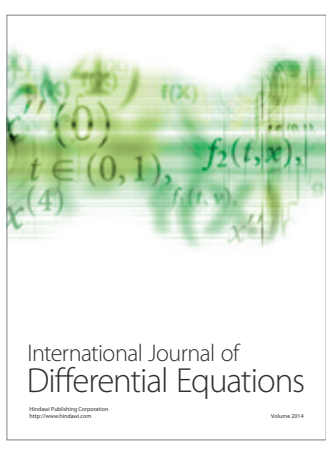
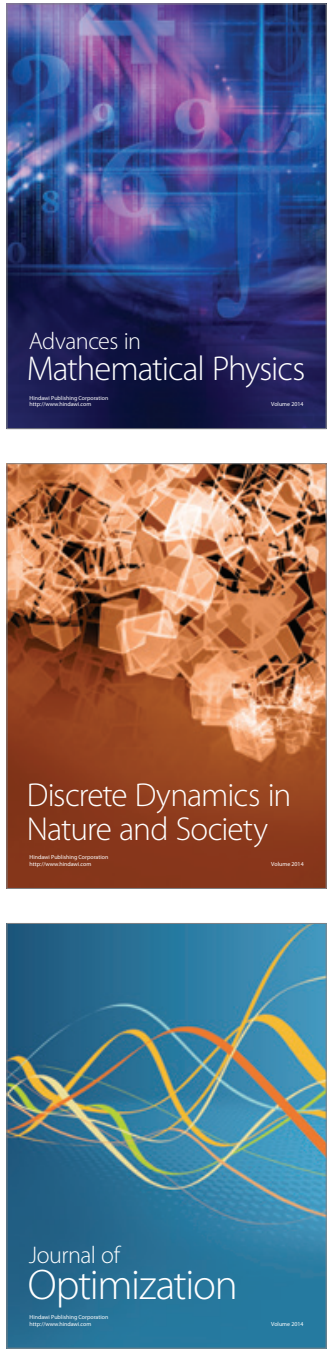